\title{
Hamiltonian dynamics with a weak noise and the echo effect for the rotator model
}

\author{
Giorgio Turchetti ${ }^{a}$ Gabriele Bassi ${ }^{\mathrm{b}}$ Armando Bazzani ${ }^{\mathrm{a}}$ \\ Bruno Giorgini ${ }^{a}$ Helmut Mais ${ }^{\mathrm{c}}$ \\ ${ }^{a}$ Dept. of Physics University of Bologna, INFN Sezione di Bologna \\ ${ }^{\mathrm{b}}$ Dept. of Mathematics and Statistics University of New Mexico, Albuquerque \\ ${ }^{\mathrm{c}}$ DESY Hamburg
}

\begin{abstract}
We analyze the effect of a weak noise on the Hamiltonian transport from the analytical and numerical viewpoint. A solvable model, the noisy rotator, is proposed to illustrate the basic phenomena. In the absence of noise, the phase space evolution is a shear flow, whose angular correlations decay following a power law, which depends on the smoothness of the initial action distribution. If the action has a fluctuating component, given by a Wiener process, then the angular correlations decay exponentially according to $e^{-\epsilon^{2} t^{3} / 6}$ or faster, where $\epsilon$ is the noise amplitude. The echo effect is well suited to investigate the competition between the decorrelation due to filamentation and noise. The noisy rotator model allows an exhaustive analytical investigation of the process for a wide class of initial conditions and a generic disturbance. The echo time is proportional to the delay $\tau$ of the disturbance and its amplitude is proportional to $\lambda \tau$ where $\lambda$ is the amplitude of the disturbance. The noise reduces the echo amplitude by $e^{-c \epsilon^{2} \tau^{3}}$, where $c$ depends on the Fourier components of the initial angular distribution, and of the disturbance applied at time $\tau$. The analytical results, derived in the limit $\lambda \rightarrow 0, \tau \rightarrow \infty$, with $\lambda \tau$ finite and sufficiently small to justify a first order expansion, are checked numerically. For more realistic models the analytical procedure would provide qualitative results and scaling laws. Quantitative results are obtained by solving the Fokker-Planck equation with a numerical scheme based on splitting: back propagation and biquadratic interpolation for the integrable part, implicit finite difference scheme for the noise component. The application to a noisy pendulum describing the longitudinal dynamics in a particle accelerator is considered, and we determine the value of the noise amplitude $\epsilon$, below which the echo cannot be detected.
\end{abstract}

Key words: Integrable Hamiltonians, filamentation, echo, weak noise, splitting.

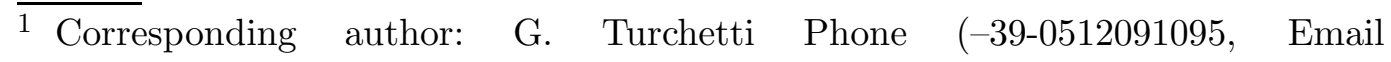

Preprint submitted to Elsevier Science $\quad 25$ March 2007 


\section{Introduction}

The integrable Hamiltonian systems with a weak noise are physically relevant, especially for beam dynamics. Indeed for proton machines the debunching effects of a small noise may cause the formation of a coasting beam. The accelerator physicists have pointed out the need of developing diagnostic tools to measure the noise level in the beam. The echo effect, first discovered in spin systems [1], and subsequently observed in solids [2] and in plasmas [3],[4], [5], [6] is a signature of coherence in a filamented phase space distribution and it is very sensitive to the noise. Therefore it can be used to detect the presence of noise in a beam and to measure its level if the relation with the echo amplitude is known. The use of the echo effect to analyze the transverse motion of the beam in a circular accelerator was first proposed by Stupakov [7], [8]. The suggested procedure consists in displacing a Gaussian beam, invariant over one turn, with a dipole kick, and to apply a quadrupole kick at time $\tau$; the echo signal appears at time $2 \tau$ in this case. The measurement of echo signals to investigate the longitudinal motion of the beam was also proposed and experimentally achieved [9][10]. The decay law of the echo amplitude in the presence of noise was established [11]. Finally the echo was proposed to measure the diffusive effects induced by space charge collective phenomena or by intrabeam scattering [12],[13]. An analysis of the echo in the general framework of dynamical systems has also been proposed [14].

We analyze the rotator model to investigate the transport in phase space and the dependence of the echo signal on the smoothness of the initial condition. The frequency is a linear function of the action and its variation causes the filamentation of any initial angle dependent distribution. This is a sort of local weak mixing and the decay of correlations follows a power law with an exponent which depends on the smoothness of the initial action distribution. However if at time $\tau$, large enough to reach an almost isotropic distribution, we apply a disturbance of small amplitude $\lambda$, then at a later time a new anisotropic signal reappears and its amplitude is proportional to $\lambda \tau$ times the frequency variation rate. This is the echo of the initial signal, which can be observed because at time $\tau$ some angular correlation still exists. If $\lambda$ is small enough, the secondary impulse anisotropy can hardly be observed and in any case it dies out following a power law in $t-\tau$. After some time proportional to $\tau$ an echo signal is produced and its amplitude is comparable with the initial distribution, if $\lambda \tau$ times the frequency variation rate is of order one.

We consider a noisy rotator whose action fluctuates according to a Wiener process. As a consequence the angle fluctuates according to the time integral of a Wiener process. The mean square deviation of the action and angle variables

turchetti@bo.infn.it. 
have a linear and a cubic growth in time respectively. Since the angle is defined on the one torus $\mathbb{T}^{1}$, its distribution becomes uniform exponentially fast, more precisely as $e^{-\epsilon^{2} t^{3} / 6}$ at least, where $\epsilon$ denotes the noise amplitude. This explains why the residual coherence, which is preserved by the slow mixing process due to filamentation, is wiped out by the presence of noise when its amplitude grows.

For the rotator model we compute the exact trigonometric moments of a wide class of distributions both in the deterministic and noisy case. An approximate analytical solution is obtained for the echo when the amplitude of the disturbance is small. Its validity is checked by comparing its trigonometric moments with the moments of the exact distribution, which satisfies the Liouville equation in the deterministic case, the Fokker-Planck equation [16] in the noisy case.

Even though the noisy rotator model captures the qualitative features of generic integrable Hamiltonians with a small stochastic perturbation, numerical procedures are needed for the quantitative analysis of realistic physical models. The numerical integration of the Fokker Planck equation associated to a Hamiltonian system with small noise is a non trivial task, since the dynamics is initially dominated by the Lagrangian transport, and a high accuracy is needed to resolve the phase space filamentated distribution. The Monte Carlo method (Langevin evolution of $N$ initial randomly generated points) is simple to use, but requires large statistics to resolve small scales $\ell$, since the relative error on the distribution is of order $1 /(\ell \sqrt{N})$, (working on a unit box partitioned into cells of side $\ell$ ). To resolve a $10^{-3}$ scale is already so expensive that it is convenient to solve the Fokker-Planck equation by using a splitting method based on the Hamiltonian transport, followed by the computation of the noise induced diffusion at every time step. The transport is achieved by the back propagation of the distribution function, which solves the Liouville equation. A symplectic integration is used to approximate the exact flow and the biquadratic interpolation, suggested by Warnock and Ellison [15], is used to evaluate the distribution at the grid points. This is a good compromise between accuracy and computational complexity. The noise induced diffusion is determined by a stable solver of the Fokker Planck equation. An alternative approach is provided by the stochastic Liouville equation [17], which consists in computing the density function at time $t$ for a given initial distribution and a given realization of the noise. The standard p.d.f. (probability density function) solution of the Fokker-Planck equation is then obtained by averaging the distributions obtained for different realizations. If we consider a limited number of points in a small region, this method provides a very accurate p.d.f. since we can choose a large number $N$ of realizations of the noise. We recall that while solving the Fokker Planck equation numerically, the p.d.f. has to be computed at all the grid points.

As an application of physical interest we consider a pendulum like Hamilto- 
nian describing the RF cavities of HERA-p [20]. The Monte-Carlo solution is compared with the p.d.f. obtained on the grid from the splitting method and with the p.d.f. computed as the average of the density satisfying the stochastic Liouville equation. The second method appears to provide an adequate global description of the problem on the whole phase space, whereas the last one can be used as a magnifying glass to explore small regions, otherwise unaccessible, due to insufficient resolution.

The plan of the paper is the following. In section 1 we consider the rotator model and a class of invariant initial conditions. In section 2 we examine the decoherence of a distribution due to filamentation, and its time scale $t_{\mathrm{dec}}$. In section 3 we examine the echo effect triggered by a small disturbance at time $\tau \gg t_{\text {dec }}$. In section 4 we consider an integrable Hamiltonian with a generic noise showing that it can be reduced to a rotator with additive noise by an averaging method. In section 5 we analyze the decoherence of an anisotropic distribution in presence of noise. In section 6 we consider the echo effect in presence of noise and show that the trigonometric moments of the distribution are the same as in the deterministic case up to an exponentially time decreasing factor. In section 7 we compare three numerical methods to determine the transport in phase space in presence of noise: the Monte-Carlo method, the stochastic Liouville equation and the Fokker-Planck equation. In section 8 we discuss an application to the RF cavities of HERA-p.

\section{The rotator model and the initial distributions}

The system we consider is any integrable 1-dimensional Hamiltonian model $H_{0}(J)$ defined on the cylinder $\mathbb{T} \times \mathbb{R}$. This is the case of the rotator, where $J$ is proportional to the angular momentum which can take positive or negative values. The dynamics of anharmonic oscillators can also be described by a Hamiltonian $H_{0}(J)$, but the action variable is defined on the positive real axis $\mathbb{R}_{+}$. Indeed the Hamiltonian in normal coordinates $X, P$ is given by $H_{0}\left(\frac{P^{2}+X^{2}}{2}\right)$ and becomes $H_{0}(J)$ after the transformation to the action angle coordinates $\Theta, J$ given by $X=\sqrt{2 J} \cos \Theta$ and $P=-\sqrt{2 J} \sin \Theta$. The transformation is invertible, since the Jacobian is 1 , but has a square root singularity at $J=0$. The use of complexified variables allows to overcome this restriction, but this extension is beyond the scope of this paper.

Without loss of generality we consider the Hamiltonian

$$
H_{0}(J)=\omega_{0} J+\omega_{1} \frac{J^{2}}{2}
$$


The initial action distribution $\Phi(J)$ defined by (2) has support on $\left[J_{*},+\infty[\right.$, a maximum at $J=J_{*}+\alpha^{-1}$, a half width $\sigma=\alpha^{-1} n^{-1 / 2}$ and its limit for $n \rightarrow \infty$ or $\alpha \rightarrow \infty$ is $\delta\left(J-J_{*}\right)$. Our choice of initial action distributions covering the entire regularity spectrum is not a matter of mathematical sophistication, but rather a necessary tool to explore the time development of the decoherence process when an angular dependence is introduced.

\section{Decoherence}

In order to investigate the decoherence effects we consider an initial angle dependent distribution

$$
\rho_{0}(\Theta, J)=\Phi(J)\left(f_{0}+f(\Theta)\right) \quad f_{0}=\frac{1}{2 \pi}
$$

where $f$ is a periodic function with zero mean such that $f_{0}+f(\Theta)>0$.

In the physically relevant case of a beam in the smooth focusing approximation, we have a nonlinear oscillator Hamiltonian and an invariant initial condition $\rho_{0}\left(X_{0}, P_{0}\right)=(2 \pi)^{-1} \Phi\left(\frac{X_{0}^{2}+P_{0}^{2}}{2}\right)$. The angle dependence is introduced by translating the distribution with a dipole kick corresponding to the map $X=X_{0}, P=P_{0}+\epsilon$. After the kick the distribution written in action angle variables defined by $X=\sqrt{2 J} \cos \Theta, P=-\sqrt{2 J} \sin \Theta$ explicitly reads

$$
\rho_{0}(\Theta, J)=(2 \pi)^{-1}\left[\Phi(J)+\epsilon(2 J)^{1 / 2} \Phi^{\prime}(J) \sin \Theta\right]+O\left(\epsilon^{2}\right) .
$$

For a Gaussian beam in $(X, P)$ coordinates we have $\Phi(J)=\sigma^{-2} \varphi_{0}\left(J / \sigma^{2}\right)$. The major difference with respect to (4) is the presence of the derivative $\Phi^{\prime}(J)$, which lowers the smoothness from $C^{n-1}$ to $C^{n-2}$. To avoid unessential complications we shall always refer to the initial condition (4) from now on. The case of a beam whose displacement $\epsilon$ due a dipole kick is comparable or large with respect to its width $\sigma$ deserves a special analytical treatment and will not be considered. The evolution at time $t$ is given by

$$
\rho(\Theta, J, t) \equiv \rho_{0}\left(\Theta_{0}, J_{0}\right)=\rho_{0}(\Theta-\Omega(J) t, J) \quad \Omega(J)=\omega_{0}+\omega_{1} J
$$

The filamentation effect, whose final result is the destruction of the signal coherence, can be observed by averaging over $J$ at time $t$. To this end we expand the angular distribution into a Fourier series and denote by $\rho_{1}(\Theta, t)$ its average with respect to $J$, by $\rho_{2}(J, t)$ its average with respect to $\Theta$.

$$
\rho_{1}(\Theta, t)=f_{0}+\sum_{k \neq 0} f_{k} e^{i k\left(\Theta-\omega_{0} t\right)} \int_{-\infty}^{+\infty} \Phi(J) e^{-i k \omega_{1} t J} d J
$$


The integral is the characteristic function $\hat{\Phi}$ of the distribution evaluated at $k \omega_{1} t$. If $\Phi(J)$ is given by (2), changing the integration variable to $x=\alpha(J-$ $\left.J_{*}\right)$, we obtain for any $n \geq 0$

$$
\rho_{1}(\Theta, t)=f_{0}+\sum_{k \neq 0} f_{k} \frac{e^{i k\left(\Theta-\Omega\left(J_{*}\right) t\right)}}{\left(1+i \frac{k \omega_{1} t}{\alpha n_{+}}\right)^{n+1}} \quad n_{+}=\left\{\begin{array}{ll}
n & \text { if } n \geq 1 \\
1 & \text { if } n=0
\end{array} .\right.
$$

If the initial action distribution is a Gaussian (3), the decoherence is exponentially fast

$$
\rho_{1}(\Theta, t)=f_{0}+\sum_{k \neq 0} f_{k} e^{i k\left(\Theta-\Omega\left(J_{*}\right) t\right)} e^{-k^{2} \omega_{1}^{2} \sigma^{2} t^{2} / 2} .
$$

The decoherence time of the distribution (4) is defined by

$$
t_{\mathrm{dec}}=\frac{1}{\left|\omega_{1}\right| \sigma}
$$

where $\sigma$ is the half width of the signal. The averages of $e^{i k \Theta}$, defined by

$$
\left\langle e^{i k \Theta}\right\rangle(t)=\int_{-\infty}^{+\infty} d J \int_{0}^{2 \pi} d \Theta e^{i k \Theta} \rho(\Theta, J, t)=\int_{0}^{2 \pi} d \Theta e^{i k \Theta} \rho_{1}(\Theta, t),
$$

show a decay rate $\lambda(k)=\left|\left\langle e^{i k \Theta}\right\rangle\left(t_{\text {dec }}\right)\right| /\left|\left\langle e^{i k \Theta}\right\rangle(0)\right|$ which increases with $n$ and $|k|$. For the distribution of class $C^{n-1}$ defined by (2), whose width is given by $\sigma=1 /\left(\alpha \sqrt{n_{+}}\right)$we have $t_{\text {dec }}=\alpha \sqrt{n_{+}} / \omega_{1}$ so that

$$
\lambda(k)=\left(1+\frac{k^{2}}{n_{+}}\right)^{-\frac{n+1}{2}} \quad n \geq 1,
$$

whereas the decay for the Gaussian signal (3) is

$$
\lambda(k)=e^{-k^{2} / 2} .
$$

Keeping constant the width $\sigma$ of the signal, the power law decay for the distribution of class $C^{n-1}$ has an exponential limit for $n \rightarrow \infty$, the same as for the real analytic signal of the Gaussian case.

\section{The echo effect}

We consider the initially perturbed distribution which evolves up to some time $\tau$ at which a new disturbance is applied. We choose $\tau \gg t_{\text {dec }}$ so that the initial beam has filamented and has become almost uniform with respect to the angle. One would expect that at times $t \gg \tau+t_{\text {dec }}$ an almost complete decoherence has occurred once more. This is not in general the case since after some time 
a new signal with angular anisotropy reappears. The amplitude of this new signal can be appreciable even though the disturbance at time $\tau$ is quite small. This remarkable phenomenon called echo, shows the deep difference between a true stochastic decoherence of the angular variable and the decoherence due to filamentation. We choose a generic kick $K$ whose inverse is exactly the standard map $\left(\Theta_{0}, J_{0}\right)=K^{-1}(\Theta, J)$ where

$$
J_{0}=J+\lambda g(\Theta) \quad \Theta_{0}=\Theta+J+\lambda g(\Theta) .
$$

For an initial distribution given by (4) the phase space density at time $t$ after the kick given at time $\tau$ reads

$$
\rho(\Theta, J, t)=\rho_{0}\left(J_{0}, \theta_{0}\right)=\rho_{0}\left(S_{-\tau} K^{-1} S_{\tau-t}(\Theta, J)\right),
$$

where by $S_{t}$ we denote the flow of the Hamiltonian $H$. We evaluate the distribution in three steps

$$
\rho(\Theta, J, t)=\rho_{I I}\left(S_{\tau-t}(\Theta, J)\right) \quad \rho_{I I}(\Theta, J)=\rho_{I}\left(K^{-1}(\Theta, J)\right) \quad \rho_{I}(\Theta, J)=\rho_{0}\left(S_{-\tau}(\Theta, J)\right),
$$

so that

$$
\rho_{I I}(\Theta, J)=\rho_{0}(\Theta+J+\lambda g(\Theta)-\Omega(J+\lambda g(\Theta)) \tau, J+\lambda g(\Theta)) .
$$

Assuming that $\lambda$ is small we perform a first order expansion

$$
\begin{gathered}
\rho_{I I}(\Theta, J)=\rho_{0}(\Theta+J-\tau \Omega(J), J)+\frac{\partial \rho_{0}}{\partial J}(\Theta+J-\Omega(J), J) \lambda g(\Theta)- \\
-\frac{\partial \rho_{0}}{\partial \Theta}(\Theta+J-\tau \Omega(J), J) \tau \Omega^{\prime}(J) \lambda g(\Theta)+\frac{\partial \rho_{0}}{\partial \Theta}(\Theta+J-\tau \Omega(J), J) \lambda g(\Theta) .
\end{gathered}
$$

The crucial hypothesis is that $\tau \gg t_{\text {dec }}$ so that the first term on the r.h.s. has lost coherence and its average over $J$ is $f_{0}$ up to negligible corrections. Of the remaining three terms the second one is by far the largest if we assume that

$$
\tau\left|\Omega^{\prime}(J)\right|=\tau\left|\omega_{1}\right| \gg 1
$$

We recall that the slowest decay of correlations $\propto t^{-1}$ occurs for a discontinuous initial action distribution given by equation (2) for $n=0$ or by the characteristic function of an interval $\left|J-J_{*}\right| \leq \sigma$. The approximation scheme we consider requires $\lambda$ very small, $\omega_{1} \tau$ large but such that $\omega_{1} \tau \lambda$ is still small so that we can drop the terms of order $\lambda$ retaining only the terms of order $\omega_{1} \lambda \tau$. We notice that the condition $\tau \gg t_{\text {dec }}$ corresponds to $\tau\left|\omega_{1}\right| \gg 1 / \sigma$. As a consequence if the action distribution has a narrow peak of half width $\sigma<1$ the condition $\tau \gg t_{\text {dec }}$ implies that $\tau\left|\omega_{1}\right| \gg 1$. Retaining only the first and the third terms in (18) we finally have

$$
\rho(\Theta, J, t) \simeq \rho_{0}(\Theta+J-\tau \Omega-(t-\tau) \Omega(J), J)-
$$




$$
\begin{aligned}
& -\frac{\partial \rho_{0}}{\partial \Theta}(\Theta+J-\tau \Omega-(t-\tau) \Omega, J) \omega_{1} \tau \lambda g(\Theta-(t-\tau) \Omega)= \\
= & \rho_{0}(\Theta+J-t \Omega, J)-\omega_{1} \lambda \tau \Phi(J) f^{\prime}(\Theta+J-t \Omega) g(\Theta-(t-\tau) \Omega) .
\end{aligned}
$$

The final step consists in computing the average of the density with respect to $J$. The computation of the phase space average of any function of the angle is then straightforward. In order to avoid writing down a double series, we assume the initial angular anisotropy and the kick at time $\tau$ to have a single Fourier component

$$
f(\Theta)=\sin (\ell \Theta) \quad g(\Theta)=\sin (m \Theta) .
$$

Using the trigonometric identity

$$
f^{\prime} g=\frac{\ell}{2} \sin ((m+\ell) \Theta+\ell J-[(m+\ell) t-m \tau] \Omega)+\frac{\ell}{2} \sin ((m-\ell) \Theta-\ell J-[(m-\ell) t-m \tau] \Omega),
$$

and choosing as initial action distribution the smooth function $\Phi(J)$ given by (2) after a straightforward integration we find for $t>\tau \gg t_{\mathrm{dec}}$

$$
\begin{array}{r}
\rho_{1}(\Theta, t) \simeq \frac{1}{2 \pi}-\ell \omega_{1} \frac{\lambda \tau}{2} \operatorname{Im}\left\{\frac{e^{i\left[(m+\ell) \Theta+\ell J_{*}-\Omega\left(J_{*}\right)((m+\ell) t-m \tau)\right]}}{\left(1+i \frac{\omega_{1}[(m+\ell) t-m \tau]-\ell}{\alpha n_{+}}\right)^{n+1}}+\right. \\
\left.+\frac{e^{i\left[(m-\ell) \Theta-\ell J_{*}-\Omega\left(J_{*}\right)((m-\ell) t-m \tau)\right]}}{\left(1+i \frac{\omega_{1}[(m-\ell) t-m \tau]+\ell}{\alpha n_{+}}\right)^{n+1}}\right\}
\end{array}
$$

where we have replaced the $J$ projection of $\rho_{0}(\Theta+J-\Omega t, J)$, given by (8), with its constant term $f_{0}=(2 \pi)^{-1}$, since the remaining terms are negligible for $t \gg t_{\text {dec }}$. The standard case $m=2, \ell=1$ is the analogue of the initial dipolar kick followed by a quadrupolar kick at time $\tau$; in this case the echo time is $2 \tau$. Having an initial signal whose amplitude is of order one, the amplitude of the echo signal is of order $\omega_{1} \lambda \tau$. If this product is of order one our approximation (20) gives an echo signal qualitatively correct as shown by figure (2), where this is compared with a numerically exact result. If $\omega_{1} \lambda \tau \ll 1$ the approximation (20) becomes quantitatively accurate. We observe that the echo signal is given by the second term in (23) since for $t=t_{\text {echo }}$ where

$$
t_{\text {echo }}=\frac{m}{m-\ell} \tau
$$


the denominator becomes of order 1 . The denominator of the first term in (23) increases (in absolute value) with $t$ having its minimum at $t=\tau+0$ whose value is $\sim\left(\ell \tau n_{+}^{-1 / 2} / t \mathrm{dec}\right)^{n+1} \gg 1$. The denominator of the second term in (23) is comparable at $t=\tau+0$ but decreases reaching at $t=t$ echo a minimum $\sim\left(1+\ell^{2} \alpha^{-2} n_{+}^{-2}\right)^{\frac{n+1}{2}}=\left(1+\ell^{2} \sigma^{2} / n_{+} \omega_{1}^{2}\right)^{\frac{n+1}{2}}$, which is of order 1 unless the initial distribution is broad $\sigma \gg 1$. The echo occurs only if $m>\ell$ and we notice that $t_{\text {echo }}=2 \tau$ if $m=2 \ell, t_{\text {echo }} \rightarrow \tau$ if $m$ grows keeping $\ell$ fixed, whereas $t_{\text {echo }} \gg \tau$ if $m$ grows keeping $m-\ell$ fixed.

\subsection{Anharmonic oscillators and quadrupole kick}

The echo effect in beam dynamics is observed by creating an anisotropic initial distribution with a dipole kick, and by applying a subsequent quadrupole kick, which is similar to the standard map kick, once the transformation to actionangle variables is made, provided the amplitude is small. The quadrupole kick $X=X_{0}, P=P_{0}-\lambda X$ in action angle coordinates becomes

$$
J=J_{0}\left(1+\lambda \sin \left(2 \Theta_{0}\right)+\lambda^{2} \cos ^{2} \Theta_{0}\right) \quad \tan \Theta=\lambda+\tan \Theta_{0} .
$$

At first order in $\lambda$ the transformation is easily inverted and we obtain

$$
\Theta_{0}=\Theta-\frac{\lambda}{2}(\cos 2 \Theta+1)+O\left(\lambda^{2}\right) \quad J_{0}=J(1-\lambda \sin (2 \Theta))+O\left(\lambda^{2}\right) .
$$

Using this approximation the previous formulas for the echo apply up to minor straightforward changes, we do not report. To invert the transformation exactly we write $J=J_{0}(1+f(\Theta))$ after expressing $\cos ^{2} \Theta_{0}, \sin 2 \Theta_{0}$ as a function of $\tan \Theta$ and introduce the generating function $F_{3}\left(\Theta, J_{0}\right)=-J_{0}(\Theta+F(\Theta))$ where $F(\Theta)=\int_{0}^{\Theta} f(\theta) d \theta$. The desired relation is $\Theta_{0}=-\frac{\partial F_{3}}{\partial J_{0}}=\Theta+F(\Theta)$.

\subsection{Numerical results}

We consider an anisotropic initial distribution given by (2) with $J_{*}=0, \alpha=1$ and more specifically $\rho_{0}(\Theta, J)=\varphi_{n}(J)(1+\sin \Theta)(2 \pi)^{-1}$ whose time evolution is governed by the rotator Hamiltonian (1) with $\omega_{0}=1, \omega_{1}=0.2$. The width of the distribution is $\sigma=\left(n_{+}\right)^{-1 / 2}$ and according to (10) the decoherence time is $t_{\mathrm{dec}}=5\left(n_{+}\right)^{1 / 2}$. The numerically exact result, is compared with the approximation (23) for a standard map kick $g(\Theta)=\sin (2 \Theta)$ at time $\tau$. The action integrated density $\rho_{1}(\Theta, t)$ has two Fourier components $e^{i \Theta}$, which exhibits the echo signal, and $e^{3 i \Theta}$ which does not. We quote the average of $\sin \Theta$, which, up to a factor $1 / 2$, is just the amplitude of $\sin \Theta$ in the expression of $\rho_{1}(\Theta, t)$ given by equation $(23)$ for $\ell=1, m=2, \alpha=1$ and $J_{*}=0, \Omega\left(J_{*}\right)=\omega_{0}$. The 

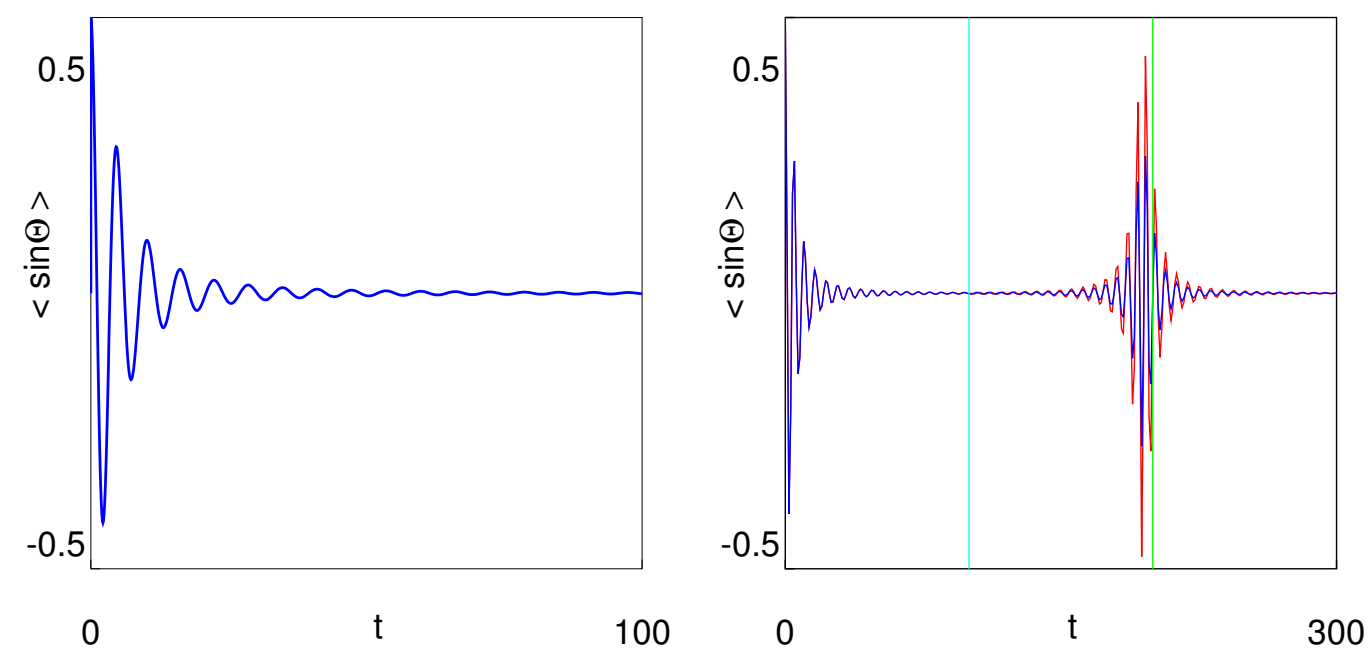

Fig. 2. Left figure: average of $\sin \Theta$ with respect to the phase space density $\rho(\Theta, J ; t)$ corresponding to a smooth initial distribution $\rho_{0}(\Theta, J)=\varphi_{3}(J)(1+\cos \Theta) /(2 \pi)$ and the flow associated to the rotator Hamiltonian (1) with $\omega_{0}=1, \omega_{1}=0.2$. The decoherence time is $t_{\mathrm{dec}} \simeq 8.66$. The right panel shows the echo effect for a standard map kick (14) of amplitude $\lambda=0.1$ and $g(\Theta)=\sin (2 \Theta)$ at time $\tau=100$. The echo is observed at time $2 \tau$. The blue line is the exact result whereas the red line corresponds to the approximation (23) leading to (27). The vertical blue line is at $t=100$, the green line at $t=200$. The exact result is obtained by (4 points repeated) Gaussian integration with 64 points in $\Theta$ and 4096 points in $J$ restricted to the interval $[0,10]$.

result explicitly reads

$$
\langle\sin \Theta\rangle=-\frac{1}{4} \omega_{1} \lambda \tau \frac{\cos \left(\omega_{0}(t-2 \tau)+\phi(t)\right)}{\left[1+\left(\frac{\omega_{1}(t-2 \tau)+1}{n_{+}}\right)^{2}\right]^{\frac{n+1}{2}}},
$$

where the phase $\phi(t)$ is given by

$$
\phi(t)=(n+1) \arctan \left(\frac{\omega_{1}(t-2 \tau)+1}{n_{+}}\right) .
$$

In figure (2) we show, for an initial signal with $n=3$, the decoherence effect by plotting $\langle\sin \Theta\rangle$ as a function of time and the echo triggered by the standard map kick of amplitude $\lambda=0.1$ at time $\tau=100$. The expansion parameter is not small $\lambda \omega_{1} \tau=2$ and consequently the agreement between the exact and the perturbative result is correct within $50 \%$ as shown by the figure, even though the shape of the echo signal is the same. Decreasing the perturbation parameter to $\lambda=0.02$, the difference between the exact and the perturbative result of the echo signal can hardly be detected within the graphics resolution, as shown by figure 3 left. 


\section{The Hamiltonian with noise}

We consider the rotator model with a small stochastic perturbation in order to examine how the decoherence due to the noise affects the echo signal. The theory developed for a multiplicative noise is applicable to the standard beam dynamics case: indeed an additive noise in Cartesian coordinates becomes multiplicative in action angle coordinates. By using the averaging theory we show that the generic problem can be reduced to a rotator with an additive noise, we can solve analytically. We start with the Hamiltonian

$$
H(\Theta, J, t)=H_{0}(J)+\epsilon H_{1}(\Theta, J) \xi(t),
$$

where $\xi(t)$ is a stochastic process that approximates the white noise in in a suitable limit. The stochastic Liouville equation for the fluctuating density $\hat{\rho}(\Theta, J, t ; \xi)$ corresponding to $(29)$ is given by

$$
\frac{\partial \hat{\rho}}{\partial t}+\Omega(J) \frac{\partial \hat{\rho}}{\partial \Theta}+\epsilon \xi(t)\left[\hat{\rho}, H_{1}\right]=0 \quad \Omega(J)=\frac{\partial H_{0}}{\partial J},
$$

where by $[A, H]_{q, p}=\frac{\partial A}{\partial q} \frac{\partial H}{\partial p}-\frac{\partial A}{\partial p} \frac{\partial H}{\partial q}$ we denote the Poisson bracket. The average of $\hat{\rho}$ with respect to the process $\xi$ is the p.d.f. $\rho$ for the stochastic Hamiltonian flow. As a consequence $\rho=\langle\hat{\rho}\rangle_{\xi}$ satisfies the Fokker-Planck equation[16] corresponding to the Stratonovich interpretation of the stochastic Hamilton's equations. This equation, which can be derived by computing the average via a Dyson expansion of the evolution operator for the fluctuating density $\hat{\rho}$ [17], explicitly reads

$$
\frac{\partial \rho}{\partial t}+\Omega \frac{\partial \rho}{\partial \Theta}=\frac{\epsilon^{2}}{2}\left[\left[\rho, H_{1}\right], H_{1}\right]
$$

The angle diffusion process is faster than action diffusion: indeed the corresponding mean square deviations are $\left\langle(\Theta-\langle\Theta\rangle)^{2}\right\rangle \sim \epsilon^{2} t^{3}$ and $\left\langle(J-\langle J\rangle)^{2}\right\rangle \sim$ $\epsilon^{2} t$. As a consequence if $t \gg \epsilon^{-2 / 3}$, the probability density $\rho$ does no longer depend on $\Theta$ and satisfies an action diffusion equation [18], [19]. Here we apply a different strategy by introducing a slow angle $\phi$ and performing a time average on a time interval, very short with respect to $\epsilon^{-2 / 3}$, on which this angle and the action do not vary appreciably. The slow angle is defined by

$$
\phi=\Theta-\Omega(J) t
$$

and the new Hamiltonian reads

$$
\epsilon \hat{H}_{1}(\phi, J, t) \xi(t)=\epsilon H_{1}(\phi+\Omega t, J) \xi(t) .
$$


The probability distribution function $\rho(\phi, J, t)$ satisfies the Fokker-Planck equation

$$
\frac{\partial \rho}{\partial t}=\frac{\epsilon^{2}}{2}\left[\left[\rho, \hat{H}_{1}\right], \hat{H}_{1}\right]
$$

corresponding to the Stratonovich interpretation of the Langevin equation associated to (33). In equation (34) [ , ] denotes the Poisson bracket. After some algebraic manipulations the equation (34) reads

$\frac{\partial \rho}{\partial \tau}=\frac{1}{2}\left(\frac{\partial}{\partial \phi}\left(\frac{\partial \hat{H}_{1}}{\partial J}\right)^{2} \frac{\partial \rho}{\partial \phi}-\frac{\partial}{\partial \phi} \frac{\partial \hat{H}_{1}}{\partial \phi} \frac{\partial \hat{H}_{1}}{\partial J} \frac{\partial \rho}{\partial J}-\frac{\partial}{\partial J} \frac{\partial \hat{H}_{1}}{\partial \phi} \frac{\partial \hat{H}_{1}}{\partial J} \frac{\partial \rho}{\partial \phi}+\frac{\partial}{\partial J}\left(\frac{\partial \hat{H}_{1}}{\partial \phi}\right)^{2} \frac{\partial \rho}{\partial J}\right)$

where we have introduced the slow time $\tau=\epsilon^{2} t$. Letting $T$ be a time interval such that $T \gg 1$ and $T \ll \epsilon^{-\alpha}$ with $\alpha<2 / 3$, we make the a priori estimate that the distribution $\rho$ remains practically constant in the time interval $[t, t+$ $T]$. By applying an averaging principle we obtain

$$
\frac{1}{T} \int_{0}^{T} \frac{\partial \rho}{\partial \tau}\left(\phi, J, \tau+\epsilon^{2} s\right) d s=\frac{\rho\left(\phi, J, \tau+\epsilon^{2} T\right)-\rho(\phi, J, \tau)}{\epsilon^{2} T} \simeq \frac{\partial \rho}{\partial \tau}(\phi, J, \tau) .
$$

From the right hand side of equation (35) we obtain the average diffusion operator. The first term is

$$
\frac{1}{T} \int_{0}^{T}\left(\frac{\partial \hat{H}_{1}}{\partial J}\right)^{2} d s \simeq \Omega^{\prime 2} t^{2} \frac{1}{T} \int_{0}^{T}\left(\frac{\partial H_{1}}{\partial \Theta}(\phi+(t+s) \Omega, J)\right)^{2} d s \simeq{\Omega^{\prime 2}}^{2}\left\langle\left(\frac{\partial H_{1}}{\partial \Theta}\right)^{2}\right\rangle_{\Theta}
$$

where $\Omega^{\prime}=d \Omega / d J$. Since $\frac{\partial \hat{H}_{1}}{\partial J}=\frac{\partial H_{1}}{\partial J}+t \Omega^{\prime}(J) \frac{\partial H_{1}}{\partial \Theta}$ we consider only the second term which is the leading one in the hypothesis $t \gg T \gg 1$ (this is correct if $H_{1}$ is a $C^{1}$ function).

In a similar way one computes

$$
\frac{1}{T} \int_{0}^{T} \frac{\partial \hat{H}_{1}}{\partial \phi} \frac{\partial \hat{H}_{1}}{\partial J} d s \simeq \Omega^{\prime} t\left\langle\left(\frac{\partial H_{1}}{\partial \Theta}\right)^{2}\right\rangle_{\Theta}
$$

and

$$
\frac{1}{T} \int_{0}^{T}\left(\frac{\partial \hat{H}_{1}}{\partial \Theta}\right)^{2} d s \simeq\left\langle\left(\frac{\partial H_{1}}{\partial \Theta}\right)^{2}\right\rangle_{\Theta}
$$

If we define

$$
\sigma^{2}(J)=\left\langle\left(\frac{\partial H_{1}}{\partial \Theta}\right)^{2}\right\rangle_{\Theta}
$$

the average Fokker Planck equation reads

$$
\frac{\partial \rho}{\partial t}=\frac{\epsilon^{2}}{2}\left(\Omega^{\prime 2} t^{2} \sigma^{2} \frac{\partial^{2} \rho}{\partial \phi^{2}}-\frac{\partial}{\partial \phi} \sigma^{2} \Omega^{\prime} t \frac{\partial \rho}{\partial J}-\frac{\partial}{\partial J} \sigma^{2} \Omega^{\prime} t \frac{\partial \rho}{\partial \phi}+\frac{\partial}{\partial J} \sigma^{2} \frac{\partial \rho}{\partial J}\right) .
$$


In the initial coordinates $\Theta, J$ the Fokker-Planck equation (41) reads

$$
\frac{\partial \rho}{\partial t}+\Omega(J) \frac{\partial \rho}{\partial \Theta}=\frac{\epsilon^{2}}{2} \frac{\partial}{\partial J} \sigma^{2}(J) \frac{\partial \rho}{\partial J}
$$

which is associated to the Langevin equation

$$
\left\{\begin{array}{l}
\dot{\Theta}=\Omega(J) \\
\dot{J}=\epsilon \sigma(J) \xi(t)
\end{array} .\right.
$$

When we consider the echo effect, the equation (41) has to be integrated for a time interval comparable with the angular diffusion time-scale $t<(\sigma \epsilon)^{-2 / 3}$. Since the variance of $J$ is of order $\epsilon^{2} t$ we can approximate in probability the fundamental solution, corresponding to initial condition $J=J_{0}$ with the solution of the equation $\dot{J}=\epsilon \sigma\left(J_{0}\right) \xi(t)$. The corresponding Fokker-Planck equation is therefore

$$
\frac{\partial \rho}{\partial t}+\Omega(J) \frac{\partial \rho}{\partial \Theta}=\frac{\epsilon^{2}}{2} \sigma^{2}\left(J_{0}\right) \frac{\partial^{2} \rho}{\partial J^{2}} .
$$

If $H_{0}(J)$ is a convex function, without loss of generality we can choose $\Omega(J)=$ $\omega_{0}+\omega_{1} J$ which corresponds to the rotator model. We remark that the angular diffusion process is faster than the action diffusion. Indeed the corresponding mean square deviations are $\left\langle\left(J-J_{0}\right)\right\rangle=\epsilon^{2} t$ and $\left\langle\left(\Theta-\Theta_{0}\right)^{2}\right\rangle=\epsilon^{2} t^{3}$ and this justifies the a priori assumption that $\rho(\phi, J, t)$ remains almost constant in the time interval $t, t+T$.

\subsection{The rotator with an additive noise}

The Langevin equation (43) with $\sigma\left(J_{0}\right)$, whose Fokker-Planck equation is (44) with $\Omega=\omega_{0}+\omega_{1} J$, corresponds to a rotator whose Hamiltonian is $H_{0}=\omega_{0} J+$ $\frac{1}{2} \omega_{1} J^{2}$ with an additive noise. Translating the actions by $\omega_{0} / \omega_{1}$ and rescaling the Hamiltonian by $\omega_{1}^{-1}$ and time by $\omega_{1}$ leaves Hamilton's equations invariant. Still denoting by $\epsilon$ the previous noise amplitude multiplied by $\sigma\left(J_{0}\right) \omega_{1}^{-1 / 2}$ the noisy rotator Hamiltonian becomes

$$
H=\frac{1}{2} J^{2}+\epsilon \xi(t) H_{1}(\Theta) \quad H_{1}(\Theta)=-\Theta \bmod 2 \pi .
$$

The corresponding stochastic Hamilton's equations are

$$
\dot{\Theta}=J \quad \dot{J}=\epsilon \xi(t)
$$

We consider first the solutions defined on the covering space $\mathbb{R}^{2}$, and subsequently wrap around the corresponding solution onto the cylinder, defined as 
the product of the one torus times the real line. Choosing the initial conditions $\Theta_{0}$ and $J_{0}$ in the interval $[0,2 \pi]$ and $\mathbb{R}$ respectively we have

$$
J=J_{0}+\epsilon w(t) \quad \Theta(t)=\Theta_{0}+J_{0} t+\epsilon \int_{0}^{t} w(s) d s
$$

The fluctuating parts of $J$ and $\Theta$ are a Wiener process and its integral respectively. The action fluctuates and its variance $\sigma_{11}$ grows as $\epsilon t^{1 / 2}$ so that at any time negative values of the action are possible. However if an initial distribution (4), with $\Phi(J)$ given by (2) peaked at $J_{*}>0$, has a small width $\sigma \ll J_{*}$, then the measure of the real negative axis $\mathbb{R}_{-}$computed from the p.d.f. at time $t$ is negligible as long as $t \ll J_{*}^{2} / \epsilon^{2}$. In this time range the model is adequate to describe the diffusion of a noisy anharmonic oscillator. Denoting by $x_{1}(t)=\Theta(t)-\Theta_{0}-J_{0} t$ and $x_{2}(t)=J(t)-J_{0}$ the fluctuating parts of the angle and action variables, the coefficients of the matrix $\sigma_{i j}^{2}(t)=\left\langle x_{i}(t) x_{j}(t)\right\rangle$, are given by

$$
\sigma_{11}^{2}(t)=\epsilon^{2} t \quad \sigma_{22}^{2}(t)=\frac{\epsilon^{2}}{3} t^{3} \quad \sigma_{12}^{2}(t)=\frac{\epsilon^{2}}{2} t^{2} .
$$

\subsection{Solutions of the Fokker-Planck equation}

Letting $A$ be the matrix whose inverse is defined by $\left(A^{-1}\right)_{i j}=2 \sigma_{i j}^{2}$ and $\mathbf{x}=\left(x_{1}, x_{2}\right)$ be the vector whose elements are the fluctuating parts of the angle and the action, the Gaussian probability density function of the process defined by equation (46) reads

$$
\rho(\Theta, J, t)=\frac{1}{\pi \sqrt{\operatorname{det} A(t)}} \exp (-\mathbf{x} A(t) \mathbf{x}),
$$

and satisfies the Fokker-Planck equation

$$
\frac{\partial \rho}{\partial t}+J \frac{\partial \rho}{\partial \Theta}=\frac{\epsilon^{2}}{2} \frac{\partial^{2} \rho}{\partial J^{2}}
$$

which is not separable. The marginal distributions $\rho_{1}(\Theta, t)$ and $\rho_{2}(J, t)$ obtained by integrating $\rho$ with respect to $\Theta$ and $J$ respectively satisfy the following equations

$$
\frac{\partial \rho_{1}}{\partial t}+J_{0} \frac{\partial \rho_{2}}{\partial \Theta}=\frac{\epsilon^{2}}{2} \frac{\partial^{2} \rho_{1}}{\partial \Theta^{2}} \quad \frac{\partial \rho_{2}}{\partial t}=\frac{\epsilon^{2}}{2} \frac{\partial^{2} \rho_{2}}{\partial J^{2}} .
$$

Bringing the distribution from $\mathbb{R}^{2}$ to the cylinder, namely rendering periodic 
its dependence in $\Theta$, we get

$$
\rho(\Theta, J, t)=\frac{\exp \left(-\frac{\left(J-J_{0}\right)^{2}}{2 \epsilon^{2} t}\right)}{\sqrt{2 \pi \epsilon^{2} t}} \times \frac{1}{2 \pi}\left(1+2 \sum_{k=1}^{\infty} e^{-\frac{1}{24} k^{2} \epsilon^{2} t^{3}} \cos k\left(\Theta-\Theta_{0}-\frac{J+J_{0}}{2} t\right)\right) .
$$

\section{Decoherence in presence of noise}

In order to evaluate the decoherence process when the filamentation and randomization simultaneously act, we consider the action average of the density $\rho$ at time $t$. We choose an initial action density $\rho_{0}$ of class $C^{n-1}$ according to (2) or a Gaussian, both peaked at $J=J_{*}$. In order to perform the computation it is convenient to introduce the following notation

$$
G_{\epsilon}(\Theta, J ; t)=\frac{e^{-\frac{J^{2}}{2 \epsilon^{2} t}}}{\sqrt{2 \pi \epsilon^{2} t}} \frac{1}{2 \pi} \sum_{k=-\infty}^{+\infty} e^{-\frac{1}{24} k^{2} \epsilon^{2} t^{3}} e^{i k \Theta}
$$

where $G_{\epsilon}(\Theta, J ; 0)=\delta(\Theta) \delta(J)$. The fundamental solution given by (52) can be written as

$$
G_{\epsilon}\left(\Theta-\Theta_{0}-\frac{J+J_{0}}{2} t, J-J_{0} ; t\right) .
$$

The fundamental solution satisfies the group property and in the limit $t=0$ becomes $\delta\left(\Theta-\Theta_{0}\right) \delta\left(J-J_{0}\right)$. As in the noiseless case we evaluate the $\Theta$ marginal distribution $\rho_{1}(\Theta, t)$ by taking the average of the p.d.f. with respect to $J$

$$
\rho_{1}(\Theta, t)=\int_{-\infty}^{+\infty} d J \int_{-\infty}^{+\infty} d J_{0} \int_{0}^{2 \pi} d \Theta_{0} G_{\epsilon}\left(\Theta-\Theta_{0}-\frac{J+J_{0}}{2} t, J-J_{0} ; t\right) \rho_{0}\left(\Theta_{0}, J_{0}\right) .
$$

The integration over $J$ is elementary and reads

$$
\int_{-\infty}^{+\infty} d J \frac{e^{-\frac{\left(J-J_{0}\right)^{2}}{2 \epsilon^{2} t}}}{\sqrt{2 \pi \epsilon^{2} t}} e^{-i \frac{k}{2} J t}=e^{-i \frac{k}{2} J_{0} t} e^{-\frac{k^{2} \epsilon^{2} t^{3}}{8}}
$$

After integrating over $\Theta_{0}$ and denoting with $f_{k}$ the Fourier coefficients of $f(\Theta)$ and choosing the initial action distribution (2) we obtain for any $n \geq 0$

$$
\rho_{1}(\Theta, t)=f_{0}+\sum_{k \neq 0} f_{k} e^{-k^{2} \epsilon^{2} t^{3} / 6} \frac{e^{i k\left(\Theta-J_{*} t\right)}}{\left(1+i \frac{k t}{\alpha n_{+}}\right)^{n+1}}
$$

where $n_{+}$is defined as in (8). The result is the same as in the deterministic case, see equation (8), with $\omega_{0}=0, \omega_{1}=1$ except for the damping factor $e^{-k^{2} \epsilon^{2} t^{3} / 6}$ 
affecting the Fourier coefficient $f_{k}$. Having chosen $\omega_{1}=1$ the decoherence time $t_{\mathrm{dec}}=\alpha n_{+}^{1 / 2}$. For the Gaussian initial condition we have $\rho=f_{0}+$ $\sum_{k \neq 0} f_{k} e^{-k^{2} \epsilon^{2} t^{3} / 6} e^{-k^{2} \sigma^{2} t^{2} / 2} e^{i k\left(\Theta-J_{*} t\right)}$ and the decay of the Fourier component $k$ at time $t_{\text {dec }}=\sigma^{-1}$ due to filamentation is $e^{-k^{2} / 2}$, the decay due to noise is $e^{-k^{2} \epsilon^{2} \sigma^{-3} / 6}$.

\section{Echo in presence of noise}

The density at time $\tau+0$ right after the standard map kick is given by

$$
\begin{gathered}
\rho(\Theta, J ; \tau+0)=\rho(\Theta+J+\lambda g(\Theta), J+\lambda g(\Theta) ; \tau-0)=\int_{-\infty}^{+\infty} d J_{0} \int_{0}^{2 \pi} d \Theta_{0} \times \\
\times G_{\epsilon}\left(\Theta+J+\lambda g(\Theta)-\frac{J+\lambda g(\Theta)+J_{0}}{2} \tau-\Theta_{0}, J+\lambda g(\Theta)-J_{0} ; \tau\right) \rho_{0}\left(\Theta_{0}, J_{0}\right) .
\end{gathered}
$$

Finally the density at $t>\tau$ integrated over the action variable $J$ is given by

$$
\rho_{1}(\Theta, t)=\int_{-\infty}^{+\infty} d J \int_{-\infty}^{+\infty} d J_{1} \int_{0}^{2 \pi} d \Theta_{1} G_{\epsilon}\left(\Theta-\frac{J+J_{1}}{2}(t-\tau)-\Theta_{1}, J-J_{1} ; t-\tau\right) \rho\left(\Theta_{1}, J_{1} ; \tau+0\right)
$$

Even though the integrations over $J$ and $J_{1}$ may be carried out exactly it is convenient to use the following approximations in evaluating $G$

$$
\begin{array}{r}
G_{\epsilon}\left(\Theta+J-\frac{J+J_{0}}{2} \tau-\Theta_{0}, J-J_{0} ; \tau\right) \simeq G_{\epsilon}\left(\Theta+J-\tau J-\Theta_{0}, J-J_{0} ; \tau\right) \simeq \\
\simeq G_{\epsilon}\left(\Theta-J \tau-\Theta_{0}, J-J_{0} ; \tau\right)
\end{array}
$$

valid for small noise amplitude $\epsilon$ and large delay time. Indeed for $\epsilon \rightarrow 0$ the fundamental solution reduces to $\delta\left(\Theta+J-\frac{1}{2}\left(J+J_{0}\right) \tau-\Theta_{0}\right) \delta\left(J-J_{0}\right)$. As a consequence the first $\delta$ argument reduces to $\Theta-J(\tau-1)-\Theta_{0} \simeq \Theta-J \tau-\Theta_{0}$ since $\tau \gg 1$.

The result is exact in the limit $\epsilon \rightarrow 0$ and $\tau \rightarrow \infty$. We shall consider this limiting case by keeping $\tau \epsilon \ll 1$ in order to analyze the echo effect. We suppose that the amplitude $\lambda$ of the kick is very small, that $\tau$ is large so that decoherence has occurred. Moreover we suppose that $\lambda \tau$ is still small so that we can expand the solution in $\lambda$ retaining only the $\lambda \tau$ term. As a consequence we can write

$$
G_{\epsilon}\left(\Theta+J+\lambda g(\Theta)-\frac{J+\lambda g(\Theta)+J_{0}}{2} \tau-\Theta_{0}, J+\lambda g(\Theta)-J_{0} ; \tau\right) \simeq
$$




$$
\begin{array}{r}
\simeq G_{\epsilon}\left(\Theta-J \tau-\lambda \tau g(\Theta)-\Theta_{0}, J+\lambda g(\Theta)-J_{0} ; \tau\right) \simeq \\
\simeq G_{\epsilon}\left(\Theta-J \tau-\Theta_{0}, J-J_{0} ; \tau\right)+\lambda \tau g(\Theta) \frac{\partial G_{\epsilon}}{\partial \Theta_{0}}\left(\Theta-\Theta_{0}-J \tau, J-J_{0} ; \tau\right) \simeq \\
\simeq G_{\epsilon}\left(\Theta-\frac{J+J_{0}}{2} \tau-\Theta_{0}, J-J_{0} ; \tau\right)+\lambda \tau g(\Theta) \frac{\partial G_{\epsilon}}{\partial \Theta_{0}}\left(\Theta-\frac{J+J_{0}}{2} \tau-\Theta_{0}, J-J_{0} ; \tau\right),
\end{array}
$$

where we have dropped in the expansion the terms of order $\lambda$ retaining only the term of order $\lambda \tau$. Inserting (61) into (58) and integrating by parts with respect to $\Theta_{0}$ we shift the derivative from $G_{\epsilon}$ to $\rho_{0}$ with no additional term since the finite factor vanishing due to the periodicity in $\Theta_{0}$. Inserting the result into (59) and integrating over $J$ we obtain the angular distribution $\rho_{1}(\Theta, t)$ that reads

$$
\begin{aligned}
& \rho_{1}(\Theta, t)=\int_{-\infty}^{+\infty} d J \int_{-\infty}^{+\infty} d J_{0} \int_{0}^{2 \pi} d \Theta_{0} G_{\epsilon}\left(\Theta-\frac{J+J_{0}}{2} t-\Theta_{0}, J-J_{0} ; t\right) \rho_{0}\left(\Theta_{0}, J_{0}\right)- \\
& -\lambda \tau \int_{-\infty}^{+\infty} d J \int_{-\infty}^{+\infty} d J_{1} \int_{-\infty}^{+\infty} d J_{0} \int_{0}^{2 \pi} d \Theta_{1} \int_{0}^{2 \pi} d \Theta_{0} G_{\epsilon}\left(\Theta-\frac{J+J_{1}}{2}(t-\tau)-\Theta_{1}, J-J_{1} ; t-\tau\right) \times \\
& \times G_{\epsilon}\left(\Theta_{1}-\frac{J_{1}+J_{0}}{2} \tau-\Theta_{0}, J_{1}-J_{0} ; \tau\right) g\left(\Theta_{1}\right) \frac{\partial \rho_{0}}{\partial \Theta_{0}}\left(\Theta_{0}, J_{0}\right) .
\end{aligned}
$$

The first contribution in the right hand side of (62) represents the "free" evolution which occurs in the absence of the kick at $t=\tau$ and was written by using the group property of the fundamental solution. The second term takes into account the kick effect and describes the echo effect. Moreover in the limit $\epsilon \rightarrow 0$ we recover the result of the noiseless case. Recalling that $\rho=\left(f_{0}+f(\Theta)\right) \Phi(J)$ and denoting by $f_{k}$ and $g_{k}$ the Fourier components of $f(\Theta)$ and $g(\Theta)$ the integration over the angular variables gives

$$
\begin{aligned}
\rho_{1}(\Theta, t) & =\rho_{1}^{(0)}(\Theta, t)-\lambda \tau \sum_{k, k^{\prime}=-\infty}^{+\infty} e^{i k \Theta} \int_{-\infty}^{+\infty} d J_{0} \Phi\left(J_{0}\right) C_{k, k^{\prime}}\left(J_{0} ; t, \tau\right) \times \\
& \times \frac{1}{(2 \pi)^{2}} \int_{0}^{2 \pi} d \Theta_{1} \int_{0}^{2 \pi} d \Theta_{0} e^{-i\left(k-k^{\prime}\right) \Theta_{1}} e^{-i k^{\prime} \Theta_{0}} g\left(\Theta_{1}\right) f^{\prime}\left(\Theta_{0}\right),
\end{aligned}
$$

where by $\rho_{1}^{(0)}(\Theta, t)$ we denote the unperturbed term $(\lambda=0)$ given by the first term in the r.h.s of equation (62) or (55) whose explicit expression is given by equation (57) for the initial condition (4). The function $C_{k, k^{\prime}}\left(J_{0} ; t, \tau\right)$ 
is defined by

$$
\begin{gathered}
C_{k, k^{\prime}}\left(J_{0} ; t, \tau\right)=e^{-\epsilon^{2} k^{2} \frac{(t-\tau)^{3}}{24}} e^{-\epsilon^{2} k^{\prime 2} \frac{\tau^{3}}{24}} \int_{-\infty}^{+\infty} d J \int_{-\infty}^{+\infty} d J_{1} \frac{e^{-\frac{\left(J-J_{1}\right)^{2}}{2 \epsilon^{2}(t-\tau)}}}{\sqrt{2 \pi \epsilon^{2}(t-\tau)}} \frac{e^{-\frac{\left(J_{1}-J_{0}\right)^{2}}{2 \epsilon^{2} \tau}}}{\sqrt{2 \pi \epsilon^{2} \tau}} \times \\
\times e^{-i k \frac{J+J_{1}}{2}(t-\tau)} e^{-i k^{\prime} \frac{J_{1}+J_{0}}{2} \tau}=e^{-i k(t-\tau) J_{0}-i k^{\prime} \tau J_{0}} A_{k, k^{\prime}}(t, \tau),
\end{gathered}
$$

where

$$
A_{k, k^{\prime}}(t, \tau)=\exp \left\{-\frac{\epsilon^{2}}{6}\left[k^{2}(t-\tau)^{3}+k^{\prime 2} \tau^{3}+3 k^{2} \tau(t-\tau)^{2}+3 k k^{\prime} \tau^{2}(t-\tau)\right]\right\}
$$

We observe that $A_{k, k^{\prime}}=A_{-k,-k^{\prime}}$ and it can be proved that for $t \geq \tau$ the expression within the square brackets is positive even when $k k^{\prime}<0$. We make the following choice for the angular functions

$$
f(\Theta)=\sin (\ell \Theta) \quad g(\Theta)=\sin (m \Theta),
$$

and, after performing the angular integration, from the second line of equation (63) we obtain $\ell /(4 i)\left[\delta_{k^{\prime}, \ell}\left(\delta_{k, m+\ell}-\delta_{k, \ell-m}\right)+\delta_{k^{\prime},-\ell}\left(\delta_{k, m-\ell}-\delta_{k,-m-\ell}\right)\right]$. Taking into account the relation $A_{-k,-k^{\prime}}=A_{k, k^{\prime}}$, we finally obtain

$$
\begin{gathered}
\rho_{1}(\Theta, t)=\rho_{1}^{(0)}(\Theta, t)-\frac{\tau \lambda \ell}{2} A_{m+\ell, \ell}(t, \tau) \operatorname{Im}\left\{e^{i \Theta(m+\ell)} \int_{-\infty}^{+\infty} d J_{0} e^{-i J_{0}((m+\ell) t-m \tau)} \Phi\left(J_{0}\right)\right\}- \\
-\frac{\tau \lambda \ell}{2} A_{m-\ell,-\ell}(t, \tau) \operatorname{Im}\left\{e^{i \Theta(m-\ell)} \int_{-\infty}^{+\infty} d J_{0} e^{-i J_{0}((m-\ell) t-m \tau)} \Phi\left(J_{0}\right)\right\} .
\end{gathered}
$$

From the final expression it is evident that the echo signal is the same as in the noiseless case, see equation (23), except for the attenuation factor $A_{m-\ell,-\ell}(t, \tau)$ depending on the noise amplitude.

For instance in the standard case $m=2, \ell=1$ the attenuation factor in the second term is $A_{1,-1}(t, \tau)=e^{-\frac{\epsilon^{2}}{6}\left[2(t-\tau)^{3}-(t-2 \tau)^{3}\right]}$ fort $t>\tau$ and at the echo time $t=2 \tau$ it becomes $A_{1,-1}(\tau, \tau)=e^{-\epsilon^{2} \tau^{3} / 3}$. In figure 3 we compare the echo for the same parameters as in figure 2 except for the amplitude of the standard map kick we choose $\lambda=0.02$ so that the approximation made is accurate, as shown by the left panel. By choosing $\epsilon=10^{-3}$ and $\epsilon=\sqrt{6} 10^{-3}$ the echo amplitude is reduced by $e^{-1 / 3} \sim 72 \%$ and $e^{-2} \sim 13 \%$ respectively. ${ }^{2}$

$\overline{2}$ There is a slight difference apart from the attenuation factor $A_{k, k^{\prime}}(t, \tau)$ in $(66)$ with respect to (23). Indeed in (23) for $\Omega(J)=J$ the argument of the exponentials are $(m \pm \ell) \Theta-J_{*}[(m \pm \ell) t-m \tau-\ell]$ whereas in (66) after integration over $J_{0}$ the last $\ell$ is missing. Similarly in the denominators of $(23)$ we have $(m \pm \ell) t-m \tau-\ell$ 

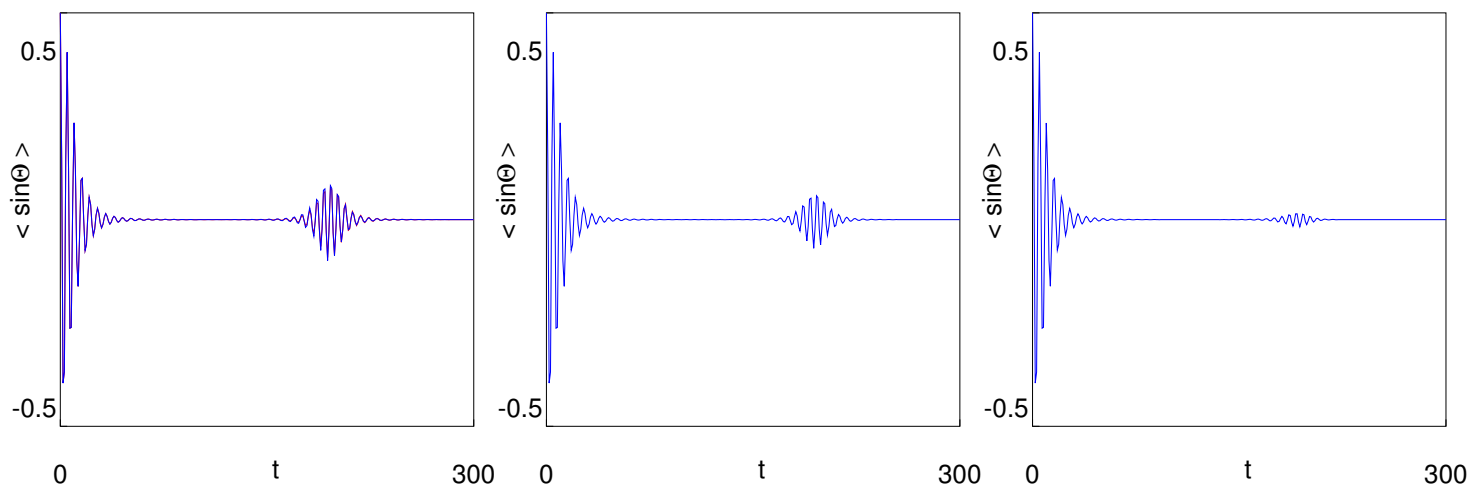

Fig. 3. Left figure: the same as figure 2 right frame for $\lambda=0.02$. Central figure: echo signal in presence of a noise of amplitude $\epsilon$ with $D=\epsilon^{2}=10^{-6}$. Right figure $D=6 \times 10^{-6}$.

\section{Numerical schemes for a generic Hamiltonian with white noise}

The transport process for a Hamiltonian system with a weak noise can be analyzed by the Monte Carlo method (MCM) sometimes denominated Brownian molecular dynamics, by the stochastic Liouville equation (SLE) or by the Fokker-Planck equation (FPE) . Given the Hamiltonian

$$
H=H_{0}(\mathbf{x}, t)+\epsilon \xi(t) H_{1}(\mathbf{x})
$$

where $\mathbf{x}$ is a point in the phase plane and $\xi(t)$ is a white noise, the stochastic Liouville equation reads [17]

$$
\frac{\partial \hat{\rho}}{\partial t}(\mathbf{x}, t ; \xi)+\left(D_{H_{0}}+\epsilon \xi(t) D_{H_{1}}\right) \hat{\rho}(\mathbf{x}, t ; \xi)=0
$$

where $D_{H}=[., H]$ denotes the Lie derivative expressed in terms of the Poisson bracket. The average over the stochastic process $\rho(\mathbf{x}, t) \equiv\langle\hat{\rho}(\mathbf{x}, t ; \xi)\rangle_{\xi}$ satisfies the Fokker Planck equation [16].

$$
\frac{\partial \rho}{\partial t}(\mathbf{x}, t)+D_{H_{0}} \rho(\mathbf{x}, t)=\frac{\epsilon^{2}}{2} D_{H_{1}}^{2} \rho(\mathbf{x}, t) .
$$

The procedure to derive equation (69) from (68) is outlined in reference [17].

whereas in (66) the last $\ell$ is missing. This is due to replacing $J(\tau-1)$ with $J \tau$ in (61). The discrepancy between (66) and (23) for $t=\tau$ amounts to replacing $\tau \pm 1$ with $\tau$. 


\subsection{Monte Carlo Methods}

The p.d.f. for the stochastically perturbed Hamiltonian (67) is obtained by approximating the initial density with $N$ randomly generated points and considering their time evolution according to the Langevin's equations of motion, with $N$ distinct realizations of the noise. The relative error on the density due to statistical fluctuations decreases as $N^{-1 / 2}$. We restrict the phase space to the unit square $[0,1]^{2}$ in the coordinates $x=\Theta /(2 \pi), y=J / J$ max , where $J_{\max } \gg J_{*}+\sigma$ and $J_{*}, \sigma$ are the peak position and the half width of the initial distribution. The analysis is limited to a time interval $[0, T]$ such that the action spread due to the noise is small and the measure of the unit square is 1 up to negligible corrections. In order to estimate the statistical error we consider a regular lattice formed by cells of side $\ell$. The exact measure and the average density $\rho_{i, j}$ of the cell $c_{i, j}$ are given by

$$
\mu\left(c_{i, j}\right) \equiv \ell^{2} \rho_{i, j}=\int_{c_{i, j}} \rho(x, y) d x d y .
$$

Approximating the density with $N$ randomly generated points weighted by $\rho$, we denote by $N_{i j}$ the number of points falling into the cell $c_{i j}$. We denote by $n_{i j}=\left\langle N_{i j}\right\rangle$ the average number of points falling in the cell $c_{i j}$, computable with a large number of realizations, and by $\Delta N_{i j}$ their variance, which are given by

$$
n_{i, j}=N \mu\left(c_{i, j}\right) \quad \Delta N_{i, j}=\left(n_{i, j}\right)^{1 / 2} .
$$

Having fixed the number $N$ we let $\ell$ vary from $N^{-1 / 2}$ to 1 . In the former case the fluctuation of the measure of any cell is comparable with the measure of the cell if the density varies gently on the unit square. This is the limit scale one can reach, since the noise and the signal are comparable. Each MonteCarlo generation provides a set of stochastic variables corresponding to the measures of the cells

$$
\mu_{\xi}\left(c_{i, j}\right)=\mu\left(c_{i, j}\right)+\Delta \mu\left(c_{i, j}\right) \xi_{i, j}
$$

where $\xi_{i, j}$ are random independent variables of zero mean and unit variance. The variance of the measure of a cell is given by

$$
\Delta \mu\left(c_{i, j}\right)=\frac{\Delta N_{i, j}}{N}=\frac{\ell\left(\rho_{i, j}\right)^{1 / 2}}{N^{1 / 2}} \quad \frac{\Delta \rho_{i, j}}{\rho_{i, j}}=\frac{\Delta \mu\left(c_{i, j}\right)}{\mu\left(c_{i, j}\right)}=\frac{1}{\ell N^{1 / 2}\left(\rho_{i, j}\right)^{1 / 2}} .
$$

If the distribution varies smoothly $\|\operatorname{grad} \rho\|<1$, then $\Delta \rho$ is an adequate estimate of the error on the density. In this case $\rho$ is close to 1 and the error $\Delta \rho / \rho$ is comparable to the lattice spacing if $\ell=N^{-1 / 4}$. If the gradients can reach large values, as in a filamented distribution, then the error is more conveniently defined in terms of the variance of the gradient averaged on a 
cell, see appendix A

$$
\frac{\Delta_{*} \rho_{i, j}}{\rho_{i, j}} \equiv \frac{2 \ell\left\|\Delta\langle\operatorname{grad} \rho\rangle_{c_{i, j}}\right\|}{\rho_{i, j}}=\frac{1}{\ell N^{1 / 2}\left(\rho_{i, j}\right)^{1 / 2}} \frac{\left\|\langle\operatorname{grad} \rho\rangle_{c_{i, j}}\right\|}{\rho_{i, j} / \ell} .
$$

When rapid oscillations are superimposed to a smooth background the error estimate given by (73) is adequate until we reach the oscillations scale where it has a sudden rise and the estimate (74) is more appropriate. For instance with $N=10^{6}$ and $\ell=10^{-2}$ the relative error is acceptable in the case of small gradients, but becomes of order 1 for gradients of order 10 as it is the case of figure 4 left, where the filamented structure can hardly be detected in the Monte Carlo simulation. Taking a one dimensional distribution, the statistical error decreases. Indeed letting $\rho(x)$ be the density and $\rho_{i}$ its average on a rectangle $c_{i}$ of base $\ell$ and unit height the errors on the corresponding densities are

$$
\frac{\Delta \rho_{i}}{\rho_{i}}=\frac{1}{\ell^{1 / 2} N^{1 / 2}\left(\rho_{i}\right)^{1 / 2}} \quad \frac{\Delta_{*} \rho_{i}}{\rho_{i}}=\frac{\Delta \rho_{i}}{\rho_{i}} \frac{\left\|\langle\operatorname{grad} \rho\rangle_{c_{i}}\right\|}{\rho_{i} / \ell} .
$$

Taking the average over the whole phase space the situation further improves and the error becomes $N^{-1 / 2}$ for a smoothly varying function $(\|\operatorname{grad} \rho\|<1)$, see appendix A, and $N=10^{6}$ is fully adequate. For a distribution filamented on a scale smaller than $10^{-2}$ with density jumps over 10 times the average local density, as in figure 4 left, the choice $\ell=10^{-3}$ and $N=10^{8}$ would not yet provide a sufficient accuracy to resolve the filaments.

\subsection{The stochastic Liouville equation}

In the case of a highly filamented phase space an alternative scheme consists in approximating the phase space density $\hat{\rho}(\mathbf{x}, t ; \xi)$, which satisfies the stochastic Liouville equation. Given the initial distribution for any realization of the noise we obtain a different density function at time $t$. Since the flow preserves the volumes the density at time $t$ at a point $\mathbf{x}$ is equal to the initial density at the back propagated point $\mathbf{x}_{0}$. Denoting by $S_{t, t_{0}}$ the evolution operator from $t_{0}$ to $t$ it follows that $\hat{\rho}(\mathbf{x}, t ; \xi)=\rho_{0}\left(S_{t_{0}, t} \mathbf{x}\right)$ where $\mathbf{x}=S_{t, t_{0}} \mathbf{x}_{0}$ and $S_{t, t_{0}} S_{t_{0}, t}=I$. The error on the density at time $t$ is only due to the integrator of the Langevin equation. Using a splitting method and a coordinate system where $H_{1}$ is a function of $q$ or $p$ only, the integration scheme is straightforward and the related error is controlled by the size of the integration step. To obtain the value of the p.d.f. $\rho(\mathbf{x}, t)$ it is sufficient to repeat the computation for $N$ distinct realization of the noise and to take the average. The statistical error $N^{-1 / 2}$ can be made small enough to resolve a small region highly filamented, since we can limit the calculation to a small number of points choosing a very large value of $N$. 


\subsection{The Fokker-Planck equation}

If we need to follow the evolution of the p.d.f. on the whole phase space and continuously in time then it is convenient to follow another strategy based on the solution of the Fokker-Planck equation. If $H_{0}$ and $H_{1}$ have no explicit time dependence the solution of equation (69) reads

$$
\rho(\mathbf{x}, t)=e^{-t D_{H_{0}}+t \frac{\epsilon^{2}}{2} D_{H_{1}}^{2}} \rho_{0}(\mathbf{x})
$$

and using the splitting

$$
\rho(\mathbf{x}, t)=e^{-\frac{1}{2} \Delta t D_{H_{0}}} e^{\Delta t \frac{\epsilon^{2}}{2} D_{H_{1}}^{2}} e^{-\frac{1}{2} \Delta t D_{H_{0}}} \rho(\mathbf{x}, t-\Delta t),
$$

the error is $O(\Delta t)^{3}$. Using the unsymmetrized splitting the error is $O(\Delta t)^{2}$. The deterministic evolution by $H_{0}$ is such that the value of $\rho$ on a regular grid at time $\Delta t$ is equal to the values of $\rho_{0}$ on a slightly distorted grid. An interpolation is needed to compute them from the values of $\rho_{0}$ on the same grid.

The biquadratic interpolation suggested by Warnock and Ellison, see equation (42) in [15], appears to be adequate, whereas the simple bilinear interpolation is not enough accurate. The diffusive contribution is then computed by using any stable finite difference algorithm on the grid like a Crank-Nicholson scheme. Explicit schemes are also stable when the noise amplitude is very small. After a new deterministic back propagation by $\Delta t / 2$ the desired value of $\rho(\mathbf{x}, \Delta t)$ at the grid points is obtained.

The choice of the time step $\Delta t$ is a crucial issue. Indeed to reach a time $t$ the number $m$ of required iterations affects the interpolation error which increases monotonically with $m$ and dominates the error due to backwards symplectic integration. Conversely the error due to the Fokker-Planck integration decreases monotonically with $m$. For any $t$ there appears to be an unique optimal choice of $\Delta t$ which minimizes the error [21]. Choosing a sufficiently fine grid and using an optimal integration step accurate results are obtained ( typically $t=100, \Delta t=0.1, \ell=10^{-3}$ ).

\section{HERA-p RF cavities and the noisy pendulum}

We discuss a physical application, that motivates the mathematical investigation on the decoherence and echo for the noisy oscillator model, developed in the previous sections.

In HERA two rf-systems are used (52 MHz, $208 \mathrm{MHz}$ ) and the longitudinal 

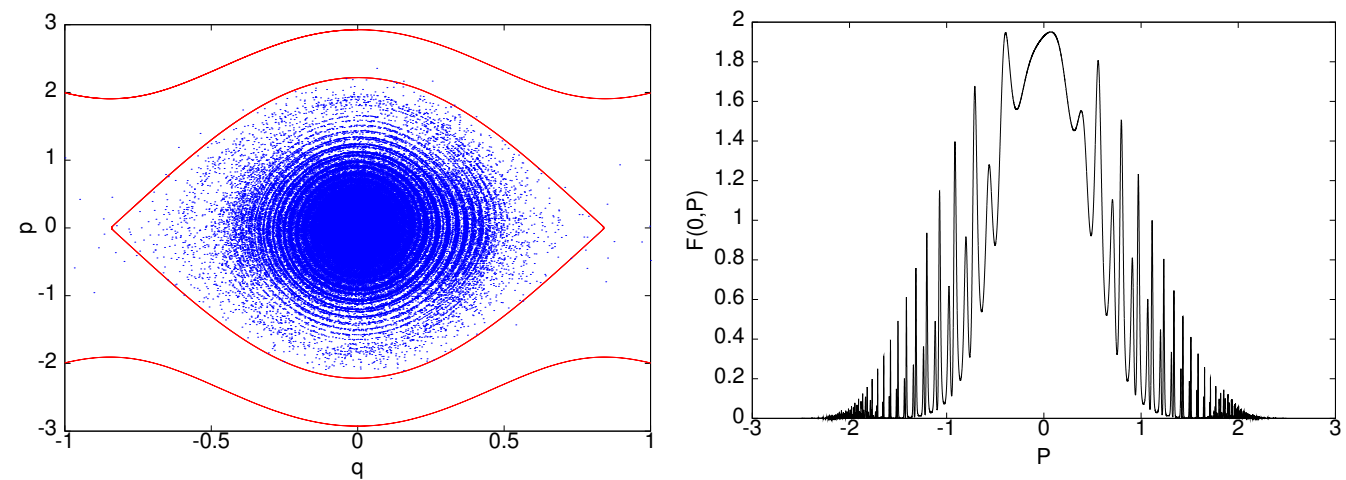

Fig. 4. Left: plot at time $t=2 \tau$ obtained with a symplectic integrator of the pendulum Hamiltonian (79), from a Monte Carlo generated initial set corresponding to a Gaussian $\rho_{0}(q, p)=0.563 \exp \left(p^{2} / 0.8+(q-0.05)^{2} / 0.4\right)$ distribution. Right: distribution on the $q=0$ line $\rho(0, p, 2 \tau)$ obtained by propagating the initial density assigned initially at $5 \times 10^{4}$ equally spaced points on the interval $-3<p<3$ of the $q=0$ line. Such a fine grid is necessary to resolve the filaments in the queues.

dynamics (synchrotron motion) of the protons is described by the Hamiltonian

$$
H=h \epsilon \frac{p^{2}}{2}-\frac{e V_{1} m_{1}}{2 \pi E_{0}} \cos \left(m_{1} q\right)-\frac{e V_{2} m_{2}}{2 \pi E_{0}} \cos \left(m_{2} q\right)
$$

where $q$ is the particle's phase deviation, $p=\left(p^{\prime}-p_{0}\right) / p_{0}$ the relative particle's momentum deviation with respect to the synchronous momentum $p_{o}$. The time derivatives are $\dot{q}=d q / d t$ and $\dot{p}=d p / d t$ with $t=2 \pi n$, where $n$ is the number of revolutions. $h=1100$ is the harmonic number, $\epsilon=0.0014$ the momentum compaction factor, $E_{0}=820 \mathrm{GeV}$ the energy of the synchronous particle, $m=1$ for the cavities at $52 \mathrm{MHz}$ and $m=4$ for the cavities at $208 \mathrm{MHz}$, $e V_{1}=100 \mathrm{KeV}$ and $e V_{2}=320 \mathrm{KeV}$ are the applied voltages.

Changing to a new set of scaled variables and by adding the noise we obtain a noisy pendulum which describes RF cavities of HERA-p. The corresponding Hamiltonian reads

$$
H=\frac{p^{2}}{2}-a_{1} \cos q-\frac{a_{2}}{4} \cos 4 q-\epsilon q \xi(t)
$$

where $a_{1}=1.2875, a_{2}=4.1201$.

The echo can also be observed in the longitudinal case applying a RF phase shift and a RF amplitude jump, which play the role of the dipole and quadrupole kicks, respectively. The longitudinal echo for the synchrotron motion of the protons in HERA is governed by the equations

$$
\dot{q}=p \quad \dot{p}=-a_{1} \sin q-a_{2} \sin 4 q
$$



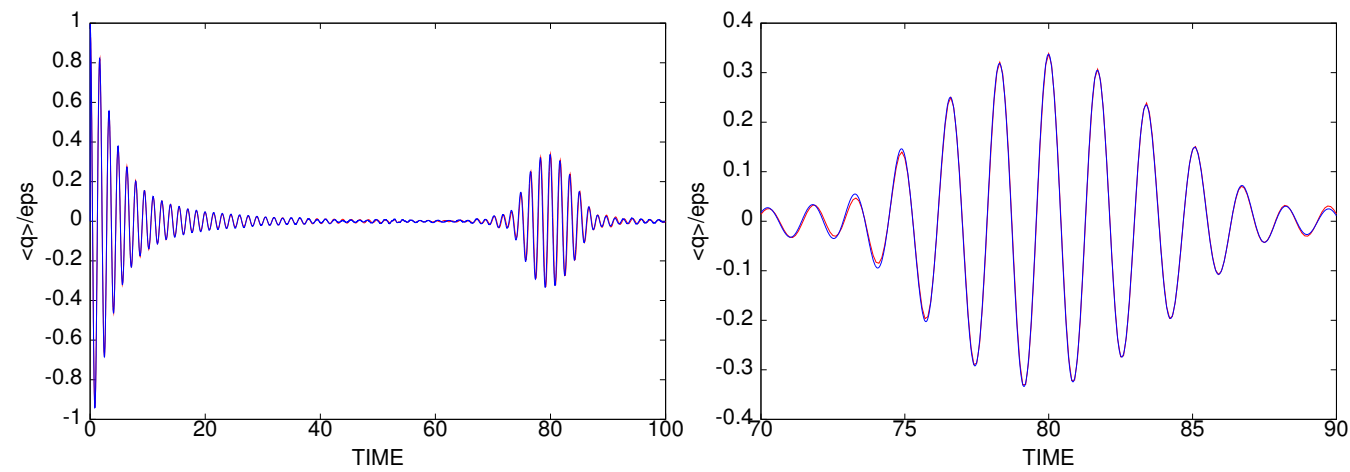

Fig. 5. Echo induced by a kick of amplitude $\lambda=0.2$ at time $\tau=40$ in absence of noise $(D=0)$. Left figure : the blue line gives the time evolution of the average $\langle q\rangle / \epsilon$ obtained with the phase space distribution resulting from a Monte-Carlo generated initial set of $10^{6}$ points; the red line gives for comparison the same average computed by solving the Liouville equation (back propagation and biquadratic interpolation) on a grid with $10^{3} \times 10^{3}$ points with $\Delta t=0.1$. Right figure: enlargement of the left figure.

As initial distribution we consider a anisotropic Gaussian translated by $\epsilon$ along the $q$ axis $\rho_{0}=\left(2 \pi \sigma_{q} \sigma_{p}\right)^{-1} \exp \left(-\frac{(q-\epsilon)^{2}}{2 \sigma_{q}^{2}}-\frac{p^{2}}{2 \sigma_{p}^{2}}\right)$ where $\sigma_{q q}=0.2, \sigma_{p p}=0.4$ and $\epsilon=0.05$. The decoherence process due to filamentation is shown in figure 4, where the evolution of the initial Gaussian distribution is computed by the Monte Carlo method and by solving the Liouville equation on a fine grid.

The initial anisotropic distribution undergoes a filamentation until a kick is applied. At time $t=\tau=40$ a kick $\lambda=0.2$ is applied in the amplitude of the first RF cavity at $52 \mathrm{Mhz}$. The choice of these parameters has been done for consistency with those used in [20].

$$
q(\tau+0)=q(\tau-0) \quad p(\tau+0)=p(\tau-0)-\lambda \sin q(\tau-0) .
$$

Due to the coherence in the filamented distribution, an echo of the signal at time $\tau$ is observed at $t=2 \tau$. The agreement between the Monte-Carlo calculation with $N=10^{6}$ points and the evolution of the distribution function based on Liouville equation, is quite good as shown by figure 5 . The effect of a small noise is shown in figures $6,7,8$, where the p.d.f. $\rho$ is obtained by solving the Fokker-Planck equation with the splitting method (back propagation on the grid for the deterministic component and finite difference for the diffusive component). By progressively increasing the noise amplitude from $\epsilon=D^{1 / 2}=$ $10^{-5 / 2}$ the distribution at time $t=2 \tau$ becomes smoother, whereas the echo signal is weakened. When $\epsilon=D^{1 / 2}=10^{-3 / 2}$ the distribution is smooth and the echo signal has disappeared. 

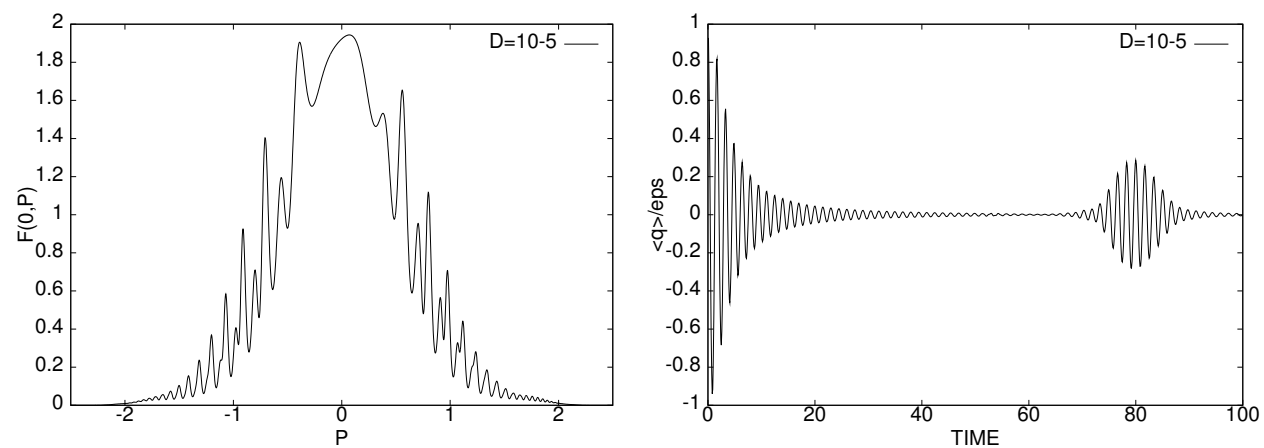

Fig. 6. Left: distribution $\rho(0, p)$ at time $2 \tau$ for the same kick and the same initial condition as figure 5 in presence of a noise with $D=\epsilon^{2}=10^{-5}$. Right: time evolution of the average $\langle q\rangle / \epsilon$.
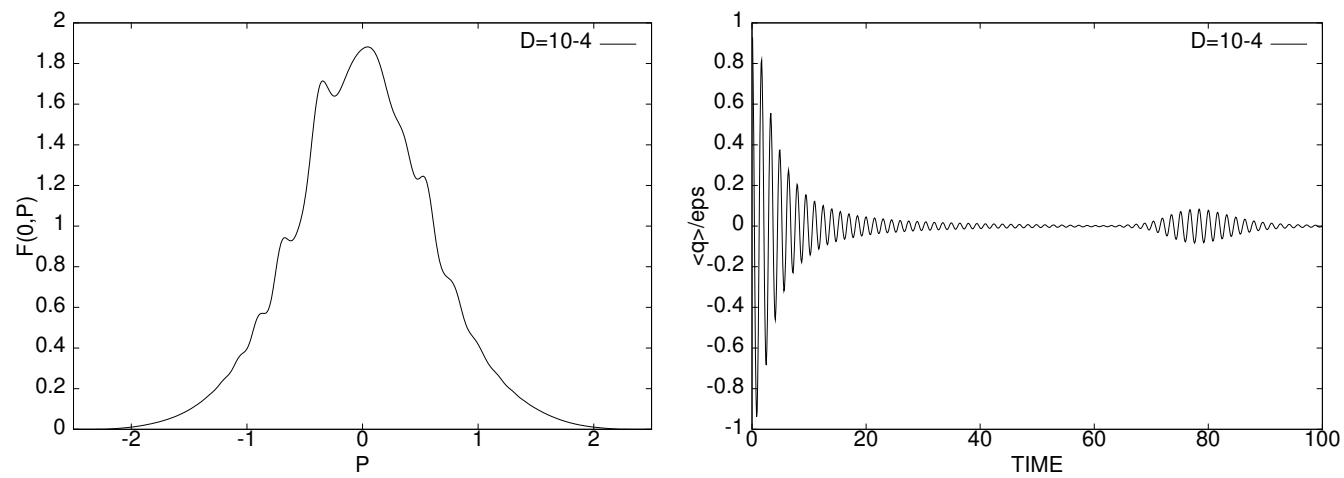

Fig. 7. The same as figure 5 for $D=10^{-4}$
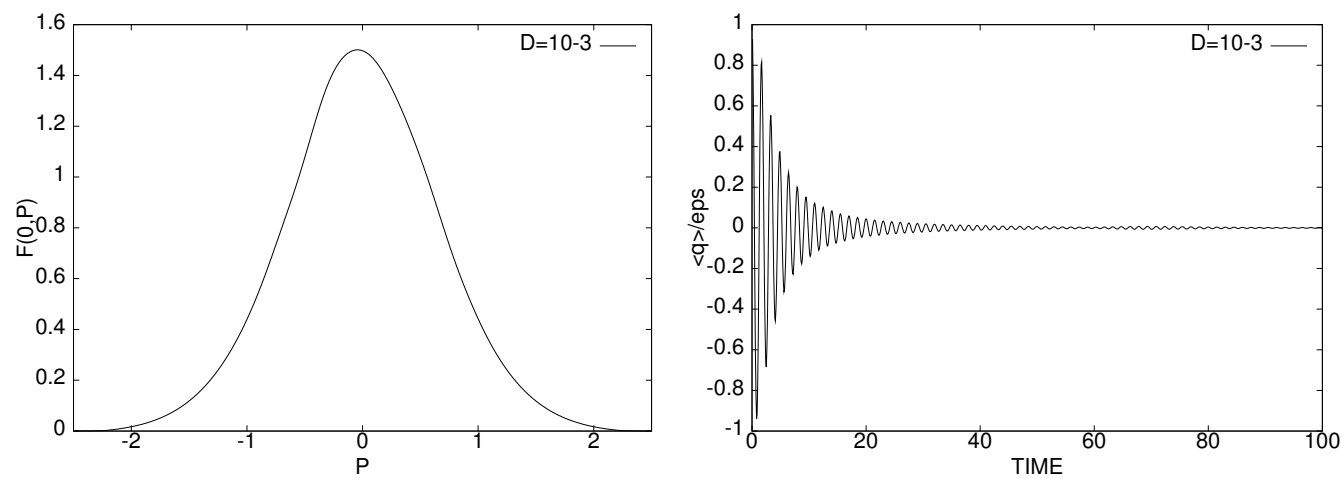

Fig. 8. The same as figure 5 for $D=10^{-3}$ 


\section{Conclusions}

We have proposed a simple Hamiltonian model, the free rotator, to analyze the effect of a weak noise on the evolution of a given initial distribution. For zero noise the phase space evolution is a shear flow on the cylinder, and the angular correlations of any initial distribution are lost due to filamentation. The decay law depends on the smoothness of the initial action distribution and varies from $t^{-1}$, for a discontinuous distribution, to $t^{-n-2}$ for smooth distributions of class $C^{n}$ to exponential for real analytic distributions like a Gaussian. The residual coherence produces the echo of any impulse given at time $\tau$. In the presence of noise the decoherence follows an exponential law and the angular correlations decay as $\exp \left(-\epsilon^{2} k^{2} t^{3} / 6\right)$ for the Fourier components $k$. If $\epsilon$ is small enough the attenuation does not prevent to observe the echo signal whose amplitude is proportional to $\lambda \tau$. In the classical dipole-quadrupole kick the amplitude of the signal at the echo time $t=2 \tau$ is $\exp \left(-\epsilon^{2} \tau^{3} / 3\right)$ times the echo amplitude in the absence of noise and it is readily seen above which level noise the echo signal disappears. The exactly solvable model applies with some caution to the anharmonic oscillator as well, provided that the support of the initial distribution is far enough from the origin. This allows to deal with the rotator dynamics defined on the cylinder $\mathbb{R} \times \mathbb{T}$, since the measure of the lower cylinder $\mathbb{R}_{-} \times \mathbb{T}$ remains negligible. For more generic integrable models having several equilibria like the pendulum or quasi integrable, such as the pendulum with a slowly and periodically varying amplitude, we have compared three different approaches based on the Monte-Carlo method, the stochastic Liouville equation and the Fokker Planck equation.

For the noisy rotator we solved analytically the Fokker Planck equation also when a standard map kick of small amplitude is applied, providing the expression of the exponential attenuation factor introduced by the noise. In order to analyze the weak noise effect on more realistic models, like a pendulum which describes the potential of a RF cavity, a numerical scheme based on a splitting method has been developed. Using the back propagation with biquadratic interpolation for the integrable unperturbed Hamiltonian and an implicit finite difference scheme to solve the Fokker-Planck equation for the noisy component, a satisfactory accuracy was obtained for discretization values requiring a few hours of CPU time to investigate the echo effect.

The Monte Carlo method is not adequate to deal with a highly filamented distribution whereas the stochastic Liouville equation allows to obtain locally the desired accuracy. The error analysis shows that the interpolation errors accumulate and increase with $(\Delta t)^{-1}$ whereas the Fokker-Planck integration error increases with $\Delta t$ so that an optimal time step is found when the errors are comparable. An application to a pendulum model with the parameters of HERA-p gives an estimate of the noise level above which the echo signal is destroyed. 
Acknowledgements A. Bazzani and G. Turchetti wish to thank DESY for hospitality during the preparation of this work. G. Bassi wishes to thank DESY for a doctoral fellowship and the US department of Energy for a partial support by Contract DE-FG03-99ER41104. This work was partially supported by a PRIN grant 2005 of the Italiam Ministery of University and research Dinamica e termodinamica di sistemi con interazioni a lungo raggio.

G. Turchetti wishes to express his gratitude to an anonymous referee for his very careful analysis of the paper and many useful remarks and suggestions. 


\section{Appendix A Statistical errors in the Monte Carlo Method}

\subsection{Error from the density gradients}

In order to give a better estimate of the statistical error when high density gradients are present we consider the average of the gradient in a cell $c_{i j}$

$$
\langle\operatorname{grad} \rho\rangle_{c_{i j}}=\left\langle\frac{\partial \rho}{\partial x}\right\rangle_{c_{i j}} \mathbf{e}_{x}+\left\langle\frac{\partial \rho}{\partial y}\right\rangle_{c_{i j}} \mathbf{e}_{y}
$$

where

$\left\langle\frac{\partial \rho}{\partial x}\right\rangle_{c_{i j}}=\frac{1}{2 \ell}\left[\int_{c_{i j}} \rho(x+\ell, y) d x d y-\int_{c_{i j}} \rho(x-\ell, y) d x d y\right]+O\left(\ell^{2}\right)=\frac{\rho_{i+1, j}-\rho_{i-1, j}}{2 \ell}+O\left(\ell^{2}\right)$.

As a consequence we obtain the statistical variance on the gradients, by using $\Delta \rho_{i j}=\ell^{-2} \Delta \mu_{i j}$ where $\Delta \mu_{i j}$ is given by $(73)$

$$
\Delta\left\langle\frac{\partial \rho}{\partial x}\right\rangle_{c_{i j}}=\frac{\Delta \rho_{i+1, j}-\Delta \rho_{i-1, j}}{2 \ell}+O\left(\ell^{2}\right)=\frac{\left(\rho_{i+1, j}\right)^{1 / 2}-\left(\rho_{i-1, j}\right)^{1 / 2}}{2 \ell^{2} N^{1 / 2}}+O\left(\ell^{2}\right) .
$$

We notice that

$$
\begin{gathered}
\left(\rho_{i+1, j}\right)^{1 / 2}-\left(\rho_{i-1, j}\right)^{1 / 2}=\left[\left(\int_{c_{i, j}} \rho(x+\ell, y) d x d y\right)^{1 / 2}-\left(\int_{c_{i, j}} \rho(x-\ell, y) d x d y\right)^{1 / 2}\right]= \\
=\frac{\ell}{\left(\rho_{i, j}\right)^{1 / 2}} \int_{c_{i, j}} \frac{\partial \rho}{\partial x}(x, y) d x d y+O\left(\ell^{3}\right)
\end{gathered}
$$

We define a new statistical variance of the density related to the variance of the gradient according to

$$
\Delta_{*} \rho_{i, j}=2 \ell\left\|\Delta\langle\operatorname{grad} \rho\rangle_{c_{i j}}\right\|=\frac{\left\|\langle\operatorname{grad} \rho\rangle_{c_{i j}}\right\|}{N^{1 / 2}\left(\rho_{i-1, j}\right)^{1 / 2}} .
$$

Finally the new relative statistical error on the density, which takes into account the rapid density variations in phase space is related to the previous one by

$$
\frac{\Delta_{*} \rho_{i, j}}{\rho_{i, j}}=\frac{1}{\ell N^{1 / 2}\left(\rho_{i, j}\right)^{1 / 2}} \frac{\left\|\langle\operatorname{grad} \rho\rangle_{c_{i j}}\right\|}{\rho_{i, j} / \ell} \equiv \frac{\Delta \rho_{i, j}}{\rho_{i, j}} \frac{\left\|\langle\operatorname{grad} \rho\rangle_{c_{i j}}\right\|}{\rho_{i, j} / \ell} .
$$


The last factor taking the gradients into account can be very large in the case of rapidly varying functions, like in filamented beam, and explains why the Montecarlo approach can fail even though for the same parameters $\ell$ and $N$ it may be adequate to describe a smooth distribution. In the case of a one dimensional distribution a similar definition holds

$$
\frac{\Delta_{*} \rho_{i}}{\rho_{i}}=\frac{1}{\ell^{1 / 2} N^{1 / 2}\left(\rho_{i}\right)^{1 / 2}} \frac{\left|\langle\partial \rho / \partial x\rangle_{c_{i}}\right|}{\rho_{i} / \ell} \equiv \frac{\Delta \rho_{i}}{\rho_{i}} \frac{\left|\langle\partial \rho / \partial x\rangle_{c_{i}}\right|}{\rho_{i} / \ell} .
$$

\subsection{Error on phase space averages}

Letting $f(x, y)$ be a smooth function such that $|\operatorname{grad} f| \leq \sqrt{2}$ and denoting by $f_{i, j}$ its value at the center of the cell $c_{i, j}$ the following estimate within the cell $\left|f(x, y)-f_{i, j}\right| \leq \ell$ holds. As a consequence the exact phase space average reads

$$
F=\int f(x, y) \rho(x, y) d x d y=\sum_{i, j} f_{i, j} \mu\left(c_{i, j}\right)+O(\ell)
$$

whereas for a Monte-Carlo sampling we have

$$
F(\xi)=\sum_{i, j} f_{i, j} \mu_{\xi}\left(c_{i, j}\right)+O(\ell)
$$

where $\mu_{\xi}\left(c_{i, j}\right)$ is given by equation (72). As a consequence by using equation (61) we readily find that the mean $\langle F(\xi)\rangle$ of the Monte-Carlo generation is $F$ up to a remainder of order $\ell$ whereas the variance is

$$
\begin{gathered}
\Delta F=\left\langle\left(F_{\xi}-F\right)^{2}\right\rangle^{1 / 2}=\left(\sum_{i, j} f_{i, j}^{2}\left(\Delta \mu\left(c_{i, j}\right)\right)^{2}+O\left(\ell^{2}\right)\right)^{1 / 2}= \\
=\left(\frac{1}{N} \sum_{i, j} f_{i, j}^{2} \mu\left(c_{i, j}\right)+O\left(\ell^{2}\right)\right)^{1 / 2} \cdot
\end{gathered}
$$

Since $\ell$ is arbitrary we can let $\ell \rightarrow 0$ and the error average of $f$ due to the Monte-Carlo sampling is

$$
\Delta F=\frac{\left\langle f^{2}\right\rangle^{1 / 2}}{N^{1 / 2}} .
$$




\section{Appendix B: Solutions of the Fokker-Planck equation}

Denoting by $\tilde{\Theta}(t)=\Theta(t)-\langle\Theta(t)\rangle$ and $\tilde{J}(t)=J(t)-\langle J(t)\rangle$ the fluctuating parts of the angle and action variables, the coefficients of the variance matrix are

$$
\begin{gathered}
\sigma_{J}^{2}(t)=\left\langle\tilde{J}^{2}(t)\right\rangle=\epsilon^{2}\left\langle w^{2}(t)\right\rangle=\epsilon^{2} t \\
\sigma_{\Theta}^{2}(t)=\left\langle\tilde{\Theta}^{2}(t)\right\rangle=\epsilon^{2}\left\langle\left(\int_{0}^{t} w(s) d s\right)^{2}\right\rangle=\frac{\epsilon^{2}}{3} t^{3}, \\
\sigma_{\Theta J}^{2}(t)=\langle\tilde{\Theta}(t) \tilde{J}(t)\rangle=\epsilon^{2}\left\langle w(t) \int_{0}^{t} w(s) d s\right\rangle=\frac{\epsilon^{2}}{2} t^{2} .
\end{gathered}
$$

We observe that the Langevin equation is linear. In this case the probability distribution $\hat{\rho}$ for the fluctuating parts is Gaussian and the distribution is given by (49) where the matrix $A$ is the inverse of the correlation matrix $2 \sigma_{i j}^{2}$

$$
A^{-1}(t)=2 \sigma^{2}(t)=\epsilon^{2}\left(\begin{array}{cc}
2 t & t^{2} \\
t^{2} & \frac{2}{3} t^{3}
\end{array}\right) \quad A=\frac{1}{\epsilon^{2}}\left(\begin{array}{cc}
\frac{2}{t} & -\frac{3}{t^{2}} \\
-\frac{3}{t^{2}} & \frac{6}{t^{3}}
\end{array}\right) .
$$

Letting $\mathbf{x}=(\hat{\Theta}, \hat{J})$ the equation satisfied by the density $\hat{\rho}(\mathbf{x}, t)$ is

$$
\frac{\partial \tilde{\rho}}{\partial t}=\frac{1}{2} \sum_{j} \frac{d \sigma_{i j}^{2}(t)}{d t} \frac{\partial^{2} \tilde{\rho}}{\partial x_{i} \partial x_{j}}
$$

As a consequence the probability density can be written as

$$
\tilde{\rho}(\tilde{\Theta}, \tilde{J}, t)=\frac{1}{\pi} \frac{\sqrt{3}}{t^{2}} \exp \left(-\frac{2}{\epsilon^{2} t} \tilde{J}^{2}+\frac{6}{\epsilon^{2} t^{2}} \tilde{\Theta} \tilde{J}-\frac{6}{\epsilon^{2} t^{3}} \tilde{\Theta}^{2}\right) .
$$

Finally the distribution in the original variables reads

$$
\rho(\Theta, J, t)=\frac{1}{\pi} \frac{\sqrt{3}}{\epsilon^{2} t^{2}} \exp \left[-\frac{\left(J-J_{0}\right)^{2}}{2 \epsilon^{2} t}\right] \exp \left[-\frac{6}{\epsilon^{2} t^{3}}\left(\Theta-\Theta_{0}-\frac{J+J_{0}}{2} t\right)^{2}\right],
$$

and satisfies the Fokker-Planck equation given by (50). 


\section{References}

[1] E.L. Hahn Phys. Rev. 80, 580 (1950)

[2] N. A. Kurnit, I. D. Abella and S. R. Hartmann Phys. Rev. Lett. 13, 567 (1964)

[3] R. M. Hill, D. E. Kaplan Phys. Rev. Lett 14, 1061 (1965)

[4] T. M. O’Neil and R. W. Gould, Phys. Fluids 11, 1 (1968).

[5] J. H. Malmberg, C. B. Wharton, R. W. Gould, and T. M. O'Neil, Phys. Rev. Lett. 20, 95 (1968).

[6] M. Porkolab a nd R. P. H. Chang, Rev. Mod. Phys. 50, 745 (1978).

[7] G. Stupakov and S. Kauffmann Echo effect in hadron colliders SSC Report SSCL-579 (1992)

[8] G. Stupakov and S. Kauffmann Echo effect in accelerators SSC Report SSCL-587 (1992)

[9] P. L. Colestock, L. K. Spentzouris, J. F. Ostiguy Proceedings of the 1995 Particle Accelerator Conference Dallas, Texas (IEEE, Piscataway, NJ, 1995) pp. 2757-2761

[10] O. Bruning, T. Linnecar, F. Ruggiero , W. Scandale , E. Shaposhnikova and D. Stellfeld, in Proceedings of the EPAC 96, Barcelona, Spain (EPAC/CERN, Geneva, 1996).

[11] A. Wu Chao, Proceedings of the U.S. particle accelerator school, Lecture Notes on topics of accelerator physics [SLAC, Stanford (unpublished)]. SLAC-PUB-9574

[12] L. K. Spentzouris, J. F. Ostiguy, P. L. Colestock Phys. Rev. Lett. 76, 620 (1996)

[13] Ahmed Al-Khateeb, Oliver Boine - Frankenhem , R. W. Hasse, and I. Hofmann Phys. Rev Spec. Topics Acc. and Beams 6, 14205 (2003)

[14] B. Eckhardt J. Physics 36 A, 371 (2003)

[15] R. L. Warnock and J. A. Ellison, Proceedings of 2nd ICFA Workshop on High Brightness Beams, UCLA, 1999 and preprint SLAC-PUB-8404 (2000)

[16] H. Risken The Fokker-Planck equation Springer Verlag (1984)

[17] G. Bassi, A. Bazzani, H. Mais, G. Turchetti Physica A 347, 17-37 (2004)

[18] A. Bazzani, S. Siboni, G. Turchetti Physica D 76,8 (1994)

[19] G. Turchetti, F. Davico Bonino Journal de Physique IV, 8, 173 (1998)

[20] E. Vogel Fast Longitudinal Diagnostics for the HERA Proton Ring PhD thesis DESY-THESIS-2002-010(2002)

[21] G. Bassi Analytical and numerical studies of stochastic effects in beam dynamics PhD thesis, Bologna University (2002) 